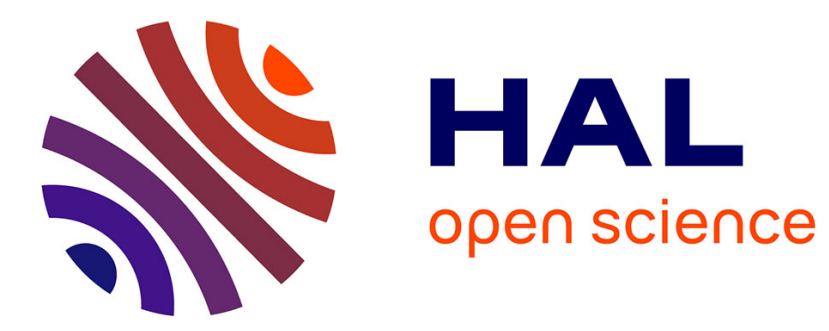

\title{
LPV system identification for control using the local approach
}

Xavier Bombois, Debarghya Ghosh, Gérard Scorletti, Julien Huillery

\section{To cite this version:}

Xavier Bombois, Debarghya Ghosh, Gérard Scorletti, Julien Huillery. LPV system identification for control using the local approach. International Journal of Control, 2019, pp.1-21. 10.1080/00207179.2019.1596316 . hal-02102192

\section{HAL Id: hal-02102192 \\ https://hal.science/hal-02102192}

Submitted on 14 May 2019

HAL is a multi-disciplinary open access archive for the deposit and dissemination of scientific research documents, whether they are published or not. The documents may come from teaching and research institutions in France or abroad, or from public or private research centers.
L'archive ouverte pluridisciplinaire HAL, est destinée au dépôt et à la diffusion de documents scientifiques de niveau recherche, publiés ou non, émanant des établissements d'enseignement et de recherche français ou étrangers, des laboratoires publics ou privés. 


\title{
LPV system identification for control using the local approach
}

\author{
X. Bombois ${ }^{\mathrm{a}, \mathrm{b}}$, D. Ghosh ${ }^{\mathrm{b}}$, G. Scorletti ${ }^{\mathrm{b}}$ and J. Huillery ${ }^{\mathrm{b}}$ \\ a Centre National de la Recherche Scientifique (CNRS), France \\ b Laboratoire Ampère, Ecole Centrale de Lyon, Université de Lyon, 36 avenue Guy de \\ Collongue, Ecully, France
}

\author{
ARTICLE HISTORY \\ Compiled February 25, 2019
}

\begin{abstract}
In this paper, we consider the problem of optimal experiment design for LPV system identification with the local approach when the identified model has to be used for LPV control. This local approach for LPV system identification is characterized by a number of local LTI identification experiments performed at constant values of the scheduling variable. In this paper, we determine these constant values of the scheduling variable as well as the input spectra of the corresponding local LTI identification experiments in an optimal way. The optimal LPV identification experiment is here defined as the experiment with the least input energy while guaranteeing that the identified LPV model is sufficiently accurate to yield a satisfactory LPV controller.
\end{abstract}

\section{KEYWORDS}

Optimal identification experiment design, LPV system identification, Identification for control

\section{Introduction}

In this paper, we consider the problem of identifying, in an optimal way, a model of a Linear Parameter Varying (LPV) system that is sufficiently accurate to design a satisfactory LPV controller for the LPV system. We thus address the problem of extending the literature on identification for control (see, e.g., [15, 19] for good surveys) to the case of LPV systems.

A LPV system is a system whose parameters vary as a function of an external variable, the so-called scheduling variable [31]. When the scheduling variable is kept constant, the LPV system reduces to a Linear Time-Invariant (LTI) system whose dynamics depend on the chosen value for the constant scheduling variable. A constant scheduling variable is often called an operating point in the literature (see, e.g., [3]).

There exist two mainstream approaches for the identification of LPV systems, namely the local [33, 23, 31, 30] and the global approaches [4, 31, 3, 22, 17]. In this paper, we will restrict attention to the so-called local approach for LPV system identification. In the first step of this local approach, the scheduling variable is kept constant at successive operating points and the corresponding local LTI models are identified using classical LTI identification [24]. In a second step, these identified models are interpolated to deliver a model of the LPV system. This second step is generally done by determining an estimate of the time-invariant coefficients $\theta^{0}$ of the (e.g. polyno- 
mial) function describing the dependence of the system parameters on the scheduling variable $[31,3]$.

In order to perform a local LPV identification experiment, a number of important choices have to me made: the operating points at which the local LTI identification experiments will be performed as well as the input spectra that will be used in each of these local LTI identification experiments. As shown, e.g., in [16], these choices strongly influence the accuracy of the identified LPV model. Consequently, it is of importance to be able to perform these choices in an optimal way. In our recent paper [16], we have determined a procedure to determine these experimental conditions in such a way that we can guarantee an user-defined model accuracy with the least input energy. In [16], the desired model accuracy was expressed in the form of a constraint $P_{\theta}^{-1}>R_{a d m}$ where $P_{\theta}$ is the covariance matrix of the estimate of $\theta^{0}$ (i.e. the size of the uncertainty region of this estimate and thus of the identified LPV model) and $R_{a d m}$ an arbitrary matrix. In the present paper, we also consider the problem of designing the LPV system identification experiment with the least input energy, but the model accuracy constraint will be now ensuring that the uncertainty of the model is small enough to guarantee that the LPV controller designed based on the identified model achieves satisfactory performance with the unknown true LPV system. As it was the case in [16], the optimal LPV identification experiment under this more complex constraint will also only be made up of a limited amount of local LTI identification experiments.

Efficient control design methods are available for LPV systems [1, 2, 29, 13]. These control design methods deliver controllers whose parameters are, such as the system itself, a function of the scheduling variable. By considering the variability of the scheduling variable as an uncertainty and by defining the performance using the $\mathcal{L}_{2}$ gain [34], robustness analysis tools have been developed to assess the performance of a closed loop made up of such LPV controller and an uncertain LPV system $[26,35$, $20,6,1]$. However, to the best of our knowledge, none of these tools tackle the type of uncertainty that follows from an LPV identification experiment. This uncertainty is indeed relatively nonstandard since it takes the form of a parametric ellipsoid centered at the estimate of $\theta^{0}$ and whose size is determined by the covariance matrix $P_{\theta}$ of this estimate. Based on our previous results on this type of nonstandard uncertainty (see, e.g., [5]), we use the separation of graph theory [28] to derive efficient robustness tools that take both this parametric uncertainty and the variability of the scheduling variable into account. Using these robustness tools, convex optimization can indeed be used to verify the robustness of a LPV controller designed based on the identified LPV model. These robustness tools can then be integrated in the optimal experiment design procedure of [16] to derive the LPV identification experiment with the least input energy that nevertheless is guaranteed to yield a model with sufficiently small uncertainty to enable robust LPV control.

It is to be noted that we will distinguish two possible situations for the way the LPV controller will be operated. In the first situation, the scheduling variable will remain constant during operation, but this constant scheduling variable can take any value in a given interval (see, e.g., [13]). This situation is referred to as the frozen aspects of an LPV representation or the Linear Parameter Invariant (LPI) situation. The second situation consists in the case that is often understood when speaking about LPV control (see, e.g., [29]), i.e., the case where the scheduling variable can vary over time during operation in a given interval. These two situations will lead to two different ways of considering the scheduling variable in the robustness analysis tools. In the second situation, the scheduling variable will have to be considered as a time-variant parametric uncertainty, while, in the first situation, the scheduling 
variable will be considered as a time-invariant parametric uncertainty.

Notations. The matrix

$$
\left(\begin{array}{ccc}
X_{1} & 0 & 0 \\
0 & \ddots & 0 \\
0 & 0 & X_{N}
\end{array}\right)
$$

will be denoted $\operatorname{diag}\left(X_{1}, \ldots, X_{N}\right)$ if the elements $X_{i}(i=1, \ldots, N)$ are scalar quantities while it will be denoted $\operatorname{bdiag}\left(X_{1}, \ldots, X_{N}\right)$ if the elements $X_{i}(i=1, \ldots, N)$ are matrices. In addition, $\mathcal{L}_{2}$ represents the set of discrete-time signals $x(t)$ with finite energy, i.e., such that $\sum_{t=0}^{\infty} x^{2}(t)<\infty$.

\section{Identification Procedure}

\subsection{True System}

We consider a Single-Input Single-Output (SISO) LPV system, i.e., a SISO system whose parameters vary with time as a function of an exogenous variable, the so-called scheduling variable $p(t)$. We assume that $p(t)$ is a scalar signal that is known at each $t$ and that varies in a given scheduling interval $\mathcal{P}$. We will further assume that the scheduling variable can be manipulated for identification purpose. Without loss of generality $^{1}$, we will define the scheduling interval $\mathcal{P}$ as:

$$
\mathcal{P}=\left[-p_{\max }, p_{\max }\right]
$$

for a given $p_{\max }$.

More precisely, we will consider an LPV system that can be described as follows:

$$
A\left(z, \zeta^{0}(p(t))\right) \breve{y}(t)=B\left(z, \zeta^{0}(p(t))\right) u(t)
$$

with $u(t)$ the input signal and $\breve{y}(t)$ the noise-free output. In $(2), A\left(z, \zeta^{0}\right)$ and $B\left(z, \zeta^{0}\right)$ are polynomials in the delay operator $z^{-1}$ and with arbitrary orders: $A\left(z, \zeta^{0}\right)=1+$ $a_{1}^{0} z^{-1}+\ldots+a_{n_{a}}^{0} z^{-n_{a}}$ and $B\left(z, \zeta^{0}\right)=z^{-n_{k}}\left(b_{0}^{0}+b_{1}^{0} z^{-1}+\ldots b_{n_{b}}^{0} z^{-n_{b}}\right)$. The vector $\zeta^{0}$ in (2) is a column vector of dimension $n \triangleq n_{a}+n_{b}+1$ containing the coefficients of these two polynomials, i.e., $a_{1}^{0}, \ldots, a_{n_{a}}^{0}, b_{0}^{0}, \ldots, b_{n_{b}}^{0}$. All the entries $\zeta_{i}^{0}(i=1, \ldots, n)$ of $\zeta^{0}$ depend on the scheduling variable $p(t)$ as follows:

$$
\zeta_{i}^{0}(p(t))=\sum_{j=0}^{n_{\zeta}} \zeta_{i, j}^{0} f_{j}(p(t)) \quad i=1, \ldots, n
$$

where $n_{\zeta}$ is an arbitrary order and $f_{j}(p(t))\left(j=0, \ldots, n_{\zeta}\right)$ correspond to a set of unisolvent and rational basis functions, e.g., $f_{j}(p(t))=p^{j}(t)$ (see, e.g., [12]). In (3), we suppose that $n_{\zeta}$ is the same for each entry $\zeta_{i}^{0}(i=$ $1, \ldots, n)$ in order to simplify the notations (this is nevertheless not a necessity). Let us define by $\theta^{0}=\left(\zeta_{1,0}^{0}, \zeta_{1,1}^{0}, \ldots, \zeta_{n, n_{\zeta}}^{0}\right)^{T}$, the vector of dimension $k \triangleq\left(n_{\zeta}+1\right) n$ which contains all the time-invariant coefficients of these polynomial expansions. The relation between $\theta^{0}$ and the time-varying parameter vector $\zeta^{0}(p(t))$

\footnotetext{
${ }^{1}$ If the scheduling space is not centered at 0 , a simple change of variable can transform it in (1).
} 
can thus be expressed as follows:

$$
\zeta^{0}(p(t))=T(p(t)) \theta^{0}
$$

for a given matrix $T(p(t))$ of dimension $n \times k$ which is only a function of $f_{j}(p(t))$ $\left(j=0, \ldots, n_{p}\right)$.

The noise-free output $\breve{y}(t)$ of $(2)$ is perturbed as follows:

$$
y(t)=\breve{y}(t)+v(t)
$$

where $v(t)$ is a stochastic signal representing both process and measurement disturbances. The signal $y(t)$ in (5) is thus the measured output of the LPV system. In the sequel, we will suppose that:

$$
v(t)=H(z) e(t)
$$

where $H(z)$ is a linear time-invariant (LTI) transfer function which is stable, inversely stable and monic and where $e(t)$ is a white noise signal of variance $\sigma_{e}^{2}$. In other words, we will suppose that $v(t)$ is independent of the scheduling variable $p$ and we will also suppose in the sequel that $H(z)$ is known. As shown in, e.g., [16], these assumptions are not required, but they strongly simplify the notations.

Based on the above description, we observe that the parameter vector $\theta^{0}$ entirely describes the LPV system. In the sequel, we will use the shorthand notation $\bar{G}\left(\zeta^{0}(p(t))\right)=\bar{G}\left(T(p(t)) \theta^{0}\right)$ for the LPV system described in (2). Using this notation, (5) can be rewritten as:

$$
y(t)=\bar{G}\left(T(p(t)) \theta^{0}\right) u(t)+v(t)
$$

where $\breve{y}(t) \triangleq \bar{G}\left(T(p(t)) \theta^{0}\right) u(t)$

\subsection{LPV system identification using the local approach}

We will now show how we can deduce an estimate $\hat{\theta}$ of $\theta^{0}$ using the local approach for LPV system identification and how we can evaluate the accuracy of this estimate $\hat{\theta}$. The first step of this local approach consists in performing a certain number (say $M$ ) of so-called local LTI identification experiments $(m=1, \ldots, M)$ [31].

Let us describe one of these local LTI identification experiments. We first bring the scheduling variable to a given constant value (or operating point) $\mathbf{p}_{\mathbf{m}}$. Then, while maintaining the scheduling variable at this operating point $p(t)=\mathbf{p}_{\mathbf{m}}$, we apply an input sequence $u(t)=u_{m}(t)\left(t=1, \ldots, N_{p}\right)$ of spectrum $\Phi_{u_{m}}$ to the LPV system (7). The duration of the experiment is thus denoted by $N_{p}$. By measuring the corresponding output $y(t)=y_{m}(t)$, we obtain the data set $Z_{m}=\left\{u_{m}(t), y_{m}(t) \mid t=1, \ldots, N_{p}\right\}^{2}$. Since $p(t)$ is kept constant to $\mathbf{p}_{\mathbf{m}}$ during the local LTI experiment, the dynamics of the true system (7) can be represented by the following LTI system described by the time-invariant parameter vectors $\zeta^{0}\left(\mathbf{p}_{\mathbf{m}}\right)=T\left(\mathbf{p}_{\mathbf{m}}\right) \theta^{0}$ :

$$
y(t)=G\left(z, \zeta^{0}\left(\mathbf{p}_{\mathbf{m}}\right)\right) u(t)+H(z) e(t)
$$

\footnotetext{
${ }^{2}$ The time index is set back to one at the beginning of each local LTI identification experiment.
} 
where $G\left(z, \zeta^{0}\left(\mathbf{p}_{\mathbf{m}}\right)\right)$ is the LTI transfer function corresponding to $\bar{G}\left(\zeta^{0}(p(t))\right)$ at the operating point $\mathbf{p}_{\mathbf{m}}$, i.e., $G\left(z, \zeta^{0}\left(\mathbf{p}_{\mathbf{m}}\right)\right) \triangleq \bar{G}\left(\zeta^{0}\left(\mathbf{p}_{\mathbf{m}}\right)\right)$. This transfer function $G\left(z, \zeta^{0}\left(\mathbf{p}_{\mathbf{m}}\right)\right)$ is therefore given by:

$$
G\left(z, \zeta^{0}\left(\mathbf{p}_{\mathbf{m}}\right)\right)=\frac{B\left(z, \zeta^{0}\left(\mathbf{p}_{\mathbf{m}}\right)\right)}{A\left(z, \zeta^{0}\left(\mathbf{p}_{\mathbf{m}}\right)\right)}=\frac{B\left(z, T\left(\mathbf{p}_{\mathbf{m}}\right) \theta^{0}\right)}{A\left(z, T\left(\mathbf{p}_{\mathbf{m}}\right) \theta^{0}\right)}
$$

with the polynomials $A(z, \zeta)$ and $B(z, \zeta)$ as defined below (2).

Based on the data set $Z_{m}$ and a full order model structure: $\mathcal{M}=\{G(z, \zeta)=$ $\left.\frac{B(z, \zeta)}{A(z, \zeta)} \mid \zeta \in \mathbf{R}^{n}\right\}$ for the LTI representation (8), we determine an estimate $\hat{\zeta}_{m}$ of $\zeta^{0}\left(\mathbf{p}_{\mathbf{m}}\right)$ using prediction error identification [24]:

$$
\hat{\zeta}_{m}=\arg \min _{\zeta} \frac{1}{N_{p}} \sum_{t=1}^{N_{p}} \epsilon_{m}^{2}(t, \zeta)
$$

with $\epsilon_{m}(t, \zeta)=H^{-1}(z)\left(y_{m}(t)-G(z, \zeta) u_{m}(t)\right)$. This estimate has the following asymptotic distribution: $\hat{\zeta}_{m} \sim \mathcal{N}\left(\zeta^{0}\left(\mathbf{p}_{\mathbf{m}}\right), P_{\zeta, m}\right)$ where the covariance matrix $P_{\zeta, m}>0$ can be estimated from the data $Z_{m}$ and $\hat{\zeta}_{m}$ [24]. Note furthermore that $P_{\zeta, m}$ has the following theoretical expression: $P_{\zeta, m}=$ $\frac{\sigma_{e}^{2}}{N_{p}}\left(\bar{E}\left(\psi_{m}\left(t, \zeta^{0}\left(\mathbf{p}_{\mathbf{m}}\right)\right) \psi_{m}^{T}\left(t, \zeta^{0}\left(\mathbf{p}_{\mathbf{m}}\right)\right)\right)\right)^{-1}$ with $\psi_{m}(t, \zeta)=-\frac{\partial \epsilon_{m}(t, \zeta)}{\partial \zeta}$ and with $\bar{E} x(t) \triangleq$ $\lim _{N_{p} \rightarrow \infty} \frac{1}{N_{p}} \sum_{t=1}^{N_{p}} E x(t)$ ( $E$ is the expectation operator) [24]. Using Parseval's theorem, we can expand this expression for $P_{\zeta, m}$ and this yields:

$$
P_{\zeta, m}^{-1}=\frac{N_{p}}{\sigma_{e}^{2}}\left(\frac{1}{2 \pi} \int_{-\pi}^{\pi} F_{u, m}\left(e^{j \omega}\right) F_{u, m}^{*}\left(e^{j \omega}\right) \Phi_{u_{m}}(\omega) d \omega\right)
$$

with $^{3} F_{u, m}(z)=\left.\frac{1}{H(z)} \frac{\partial G(z, \zeta)}{\partial \zeta}\right|_{\zeta=\zeta^{0}\left(\mathbf{p}_{\mathbf{m}}\right)}$.

The above procedure is repeated for $M$ different values of $\mathbf{p}_{\mathbf{m}}(m=1, \ldots, M)$ yielding $M$ independent estimates $\hat{\zeta}_{m}$ and their respective covariance matrix $P_{\zeta, m}$. We will assume that the durations of these $M$ local LTI experiments will be all equal to $N_{p}$, but that the spectrum $\Phi_{u_{m}}$ of the input signal can be chosen differently in these $M$ experiments. Indeed, the LTI representation (8) will be different for each $\mathbf{p}_{\mathbf{m}}$ and it makes thus sense to use a different input spectrum for these different LTI systems.

If the local LTI identication experiments have been performed at at least $n_{\zeta}+$ 1 different operating points, the estimates $\hat{\zeta}_{m}$ of $\zeta^{0}\left(\mathbf{p}_{\mathbf{m}}\right)(m=1, \ldots, M)$ can then be used to determine an estimate $\hat{\theta}$ of the time-invariant parameter vector $\theta^{0}$ using the mapping (4). Since the $M$ estimates are independent and are (asymptotically) distributed as $\hat{\zeta}_{m} \sim \mathcal{N}\left(\zeta^{0}\left(\mathbf{p}_{\mathbf{m}}\right), P_{\zeta, m}\right)$, the minimal variance estimate $\hat{\theta}$ of $\theta^{0}$ can be computed via generalized least-squares (or weighted least-squares) [16]:

$$
\hat{\theta}=\left(\sum_{m=1}^{M} T^{T}\left(\mathbf{p}_{\mathbf{m}}\right) P_{\zeta, m}^{-1} T\left(\mathbf{p}_{\mathbf{m}}\right)\right)^{-1}\left(\sum_{m=1}^{M} T^{T}\left(\mathbf{p}_{\mathbf{m}}\right) P_{\zeta, m}^{-1} \hat{\zeta}_{m}\right)
$$

and this estimate $\hat{\theta}$ has the following (asymptotic) property: $\hat{\theta} \sim \mathcal{N}\left(\theta^{0}, P_{\theta}\right)$ with a

${ }^{3}$ Note that, if the transfer function $H$ would be unknown and estimated together with $G\left(z, \zeta^{0}\left(\mathbf{p}_{\mathbf{m}}\right)\right)$, the expression (11) would remain identical [16]. 
strictly positive-definite covariance matrix $P_{\theta}$ that can be computed as [16]:

$$
P_{\theta}=\left(\sum_{m=1}^{M} T^{T}\left(\mathbf{p}_{\mathbf{m}}\right) P_{\zeta, m}^{-1} T\left(\mathbf{p}_{\mathbf{m}}\right)\right)^{-1} .
$$

This statistical property can be rewritten as $\theta^{0}-\hat{\theta} \sim \mathcal{N}\left(0, P_{\theta}\right)$ and we can therefore conclude that, with probability $\beta$, the estimation error $\theta^{0}-\hat{\theta}$ lies in the following uncertainty ellipsoid $U$ :

$$
U=\left\{\delta \theta \in \mathbf{R}^{k} \mid \delta \theta^{T} P_{\theta}^{-1} \delta \theta \leq \chi\right\}
$$

where $\operatorname{Pr}\left(\chi^{2}(k) \leq \chi\right)=\beta$. Consequently, the local approach for LPV identification not only delivers an estimate $\hat{\theta}$ for $\theta^{0}$, but also, via $P_{\theta}$ and $U$, a description of the uncertainty of this estimate $\hat{\theta}$. The estimate $\hat{\theta}$ of $\theta^{0}$ in turn defines a model $\bar{G}(T(p(t)) \hat{\theta})$ of the LPV system $\bar{G}\left(T(p(t)) \theta^{0}\right)$. The model $\bar{G}(T(p(t)) \hat{\theta})$ will be used to design a controller for $\bar{G}\left(T(p(t)) \theta^{0}\right)$.

\subsection{Some notations}

For the sequel, it will be important to note that $\bar{G}(T(p(t)) \theta)$ for any $p(t)$ and any $\theta=\hat{\theta}+\delta \theta$ is rational in both $p(t)$ and $\delta \theta$ and can therefore be written as an Linear Fractional Transformation (LFT) in $p$ and in $\delta \theta$, i.e., we can determine vectors of signals $q_{G}$ and $s_{G}$ such that the operator $\bar{G}(T(p(t)) \theta)$ between $u$ and $\breve{y}$ can be written as:

$$
s_{G}=\overbrace{\operatorname{biag}\left(p I_{n_{p_{G}}}, I_{n_{\theta_{G}}} \otimes \delta \theta\right)}^{=\Delta_{G}} q_{G} \text { and }\left(\begin{array}{c}
q_{G} \\
\breve{y}
\end{array}\right)=M_{G}(z)\left(\begin{array}{c}
s_{G} \\
u
\end{array}\right)
$$

with $I_{n_{p_{G}}}$ and $I_{n_{\theta_{G}}}$ identity matrices of given dimensions $n_{p_{G}}$ and $n_{\theta_{G}}$ and with $M_{G}(z)$ a known matrix of LTI transfer functions. Note that the transfer functions in $M_{G}$ are LTI since the time-varying element $p(t)$ has been isolated in the uncertainty block $\Delta_{G}$ of the LFT.

Moreover, the LTI transfer function $G(z, T(\mathbf{p}) \theta)=G(z, \xi(\mathbf{p}))$ corresponding to $\bar{G}(T(p(t)) \theta)$ at any given operating point $\mathbf{p}$ can be rewritten as the following rational relation in $\theta$ :

$$
G(z, T(\mathbf{p}) \theta)=\frac{Z_{N}(z, \mathbf{p}) \theta}{1+Z_{D}(z, \mathbf{p}) \theta}
$$

for some given row vectors $Z_{N}(z, \mathbf{p})$ and $Z_{D}(z, \mathbf{p})$. An example for a simple LPV system is given in Appendix A.1. 


\section{Control design based on $\bar{G}(T(p(t)) \hat{\theta})$}

\subsection{LPI and LPV control}

The control design method will generally depend on the type of scheduling sequences we will encounter during the operation of the system and the designed controller will also generally depend directly on the observed value of the scheduling variable. To stress this, we will use the following notation for the controller: $\hat{\bar{C}}(p(t))$.

In some cases, the scheduling sequence can be very close to the type of scheduling sequences considered for the local LPV identification procedure: the scheduling variable can take any value in the scheduling space $\mathcal{P}$, but remains constant during the operation of the controller. In other words, the scheduling sequences are restricted to lie in the set $\mathbf{P}$ :

$$
\mathbf{P}=\{p(t) \mid p(t)=\mathbf{p} \forall t \text { with } \mathbf{p} \in \mathcal{P}\}
$$

This is, e.g., the case when the scheduling variable $p$ represents the payload of a rocket (i.e. the mass of the to-be-launched satellites). When designing a controller for the rocket, it seems a good idea to take into account that this payload can vary from mission to mission and thus to design a controller that depends on this payload. For a given mission, the payload is nevertheless equal to a constant value $\mathbf{p}$ and the LPV model $\bar{G}(T(p(t)) \hat{\theta})$ is thus equivalent to the LTI transfer function:

$$
G(z, T(\mathbf{p}) \hat{\theta})=\frac{B(z, T(\mathbf{p}) \hat{\theta})}{A(z, T(\mathbf{p}) \hat{\theta})}
$$

which can be controlled with a LTI controller. The control problem therefore consists in determining a parametrization $\hat{C}(z, \mathbf{p})$ defining an LTI controller for each $\mathbf{p} \in \mathcal{P}$ and ensuring, for any of these values $\mathbf{p} \in \mathcal{P}$, that the closed loop $[\hat{C}(z, \mathbf{p}) G(z, T(\mathbf{p}) \hat{\theta})]$ made up of the LTI controller $\hat{C}(z, \mathbf{p})$ and the LTI model $G(z, T(\mathbf{p}) \hat{\theta})$ is stable and achieves satisfactory performance (more details will be given in the next subsection). We will call this situation Linear Parameter Invariant (LPI) control as done in [13].

In other cases, the scheduling sequence will not remain constant during the operation of the controller. In this case, control design methods yield a controller $\hat{\bar{C}}(p(t))$ whose coefficients are dependent on the time-varying scheduling sequence and that is such that the closed loop $[\hat{\bar{C}}(p(t)) \bar{G}(T(p(t)) \hat{\theta})]$ is stable and achieves satisfactory performance for all possible scheduling sequences, i.e., for all sequences $p$ lying in the set $\bar{P}$ :

$$
\bar{P}=\{p(t) \mid p(t) \in \mathcal{P} \forall t\}
$$

In this case, both the plant and the controller are time-varying operators and we will talk of LPV control.

Note that the LPI control design method can also be rephrased in a framework similar to the LPV case, i.e., as the problem of designing a controller $\hat{\bar{C}}(p(t))$ such that $[\hat{\bar{C}}(p(t)) \bar{G}(T(p(t)) \hat{\theta})]$ is stable and achieves satisfactory performance for all scheduling sequences $p \in \mathbf{P}$ (and thus not all scheduling sequences $p \in \bar{P}$ as in the LPV case). It is clear that the controller $\hat{\bar{C}}(p(t))$ designed for the LPI situation will only be used for 
$p \in \mathbf{P}$ and, from a notation point-of-view, we have that $\hat{C}(z, \mathbf{p}) \triangleq \hat{\bar{C}}(\mathbf{p}) \forall \mathbf{p} \in \mathcal{P}$.

Note finally that, in this paper, we will more particularly consider the methods of [13] for LPI control and of [29] for LPV control since the model $\bar{G}(T(p(t)) \hat{\theta})$ is rational in $p$ (see Appendix A.2. for more precision). Using these methods, the obtained controller $\hat{\bar{C}}(p(t))$ is also rational in $p$. However, any other control methods could also be considered as long as the controller can be expressed/approximated as a rational function of $p$.

Important remark. The LPV situation described above is a demanding situation. Indeed, the desired performance has to be guaranteed for scheduling variables that may vary as fast as a (bounded) white noise. In many cases, though, the scheduling variable $p(t)$ will be slowly varying and an LPI controller achieving good performance for all (frozen) operating points $\mathbf{p}$ in $\mathcal{P}$ will generally be sufficient.

\subsection{Performance requirements for $[\hat{\bar{C}}(p(t)) \bar{G}(T(p(t)) \hat{\theta})]$}

As mentioned in the previous subsection, LPI and LPV control methods such as the ones in [13, 29] allow to design, based on the model $\bar{G}(T(p(t)) \hat{\theta})$, a controller $\hat{\bar{C}}(p(t))$ guaranteeing that the closed loop $[\hat{\bar{C}}(p(t)) \bar{G}(T(p(t)) \hat{\theta})]$ (see Figure 1) is stable and exhibits a certain level of nominal performance for a given class of scheduling sequences. This level of performance can be the ability to reject certain disturbances or to track certain reference signals while maintaining an acceptable control effort. For simplicity, let us focus on one of these control aspects: the disturbance rejection ability. Let us for this purpose consider the designed loop $[\hat{\bar{C}}(p(t)) \bar{G}(T(p(t)) \hat{\theta})]$ in Figure 1 and let us denote by $\hat{\bar{S}}(p(t))$ the operator between the to-be-rejected disturbance $v$ and the output $y$, i.e., the sensitivity operator. If the plant model and the controller in Figure 1 are LTI transfer functions $(\hat{G}(z)$ and $\hat{C}(z))$, the disturbance rejection ability of the loop is classically ensured by imposing that $\left\|\hat{\bar{S}} W_{i}\right\|_{\infty} \leq \eta$ for a given scalar $\eta<1$. For this particular case, $\hat{\bar{S}}$ is an LTI transfer function, i.e., $\hat{\bar{S}}=(1+\hat{G}(z) \hat{C}(z))^{-1}$ and the weighting $W_{i}(z)$ is a (stable and inversely stable) LTI transfer function, which reflects the frequency content of the disturbance $v\left(W_{i}(z)\right.$ can be determined based ${ }^{4}$ on $H(z)$ in (6)).

In the situation considered in this paper (i.e. when both the plant and the controller can be time-varying), the performance requirements will be expressed via the notion of $\mathcal{L}_{2}$-gain [34].

Definition 1. Consider a time-varying SISO operator $\bar{L}$ that takes as input the signal $w \in \mathcal{L}_{2}$ and as output the signal $y$, i.e., $y(t)=\bar{L} w(t)$. The $\mathcal{L}_{2}$-gain $\|\bar{L}\|_{\mathcal{L}_{2}}$ of $\bar{L}$ is defined ${ }^{5}$ as:

$$
\|\bar{L}\|_{\mathcal{L}_{2}}=\sup _{w \in \mathcal{L}_{2}} \frac{\|y\|_{2}}{\|w\|_{2}}
$$

\footnotetext{
${ }^{4}$ The weighting $W_{i}(z)$ is generally determined not only based on $H(z)$, but also based on a number of other considerations (e.g. robust stability considerations).

${ }^{5} \mathrm{~A}$ limitation to signals $w$ in $\mathcal{L}_{2}$ (i.e. $w$ with finite energy) may seem restrictive. However, the quantity defined in (20) is also equal to the maximal ratio between the power of $\bar{L} w$ and the power of $w$ for any finite power signal $w$ [34].
} 


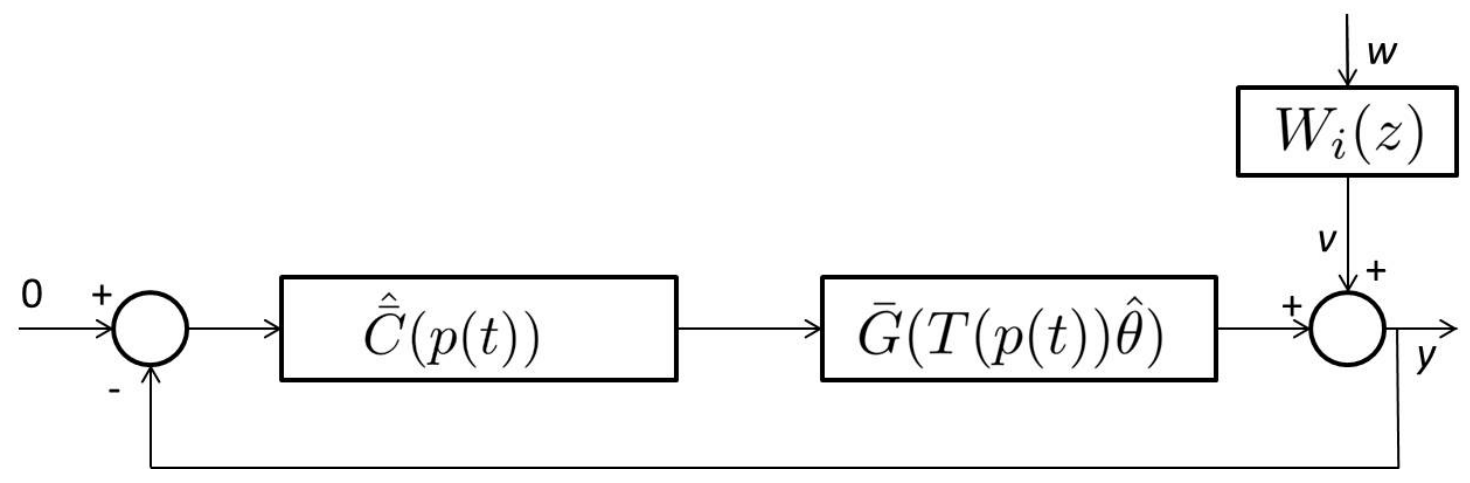

Figure 1.: The designed closed loop $[\hat{\bar{C}}(p(t)) \bar{G}(T(p(t)) \hat{\theta})]$

where $\|x\|_{2}$ is defined as:

$$
\|x\|_{2}=\sqrt{\sum_{t=0}^{\infty} x^{2}(t) .}
$$

The operator $\bar{L}$ is said stable if and only if $\|\bar{L}\|_{\mathcal{L}_{2}}<\infty$.

The fact that the $\mathcal{L}_{2}$-gain has been chosen to define performance requirements in the time-varying situation is relatively logical since the $\mathcal{L}_{2}$-gain of a LTI transfer function is its $H_{\infty}$-norm (see below for more details).

When LPV control is considered, the disturbance rejection ability of the loop in Figure 1 can be defined via the following constraint:

$$
\sup _{p \in \bar{P}}\left\|\hat{\bar{S}}(p(t)) W_{i}(z)\right\|_{\mathcal{L}_{2}} \leq \eta
$$

where $\eta$ and the weighting $W_{i}(z)$ are defined similarly as in the LTI case described above. The operator $\hat{\bar{S}}(p(t)) W_{i}(z)$ used in (22) is the operator between $w$ and $y$ in Figure 1. The weighting $W_{i}$ allows to define the class $\mathcal{V}_{W_{i}}$ of considered signals $v: \mathcal{V}_{W_{i}}$ is the class of signals $v$ such that $\left\|W_{i}^{-1} v\right\|_{2}<\|v\|_{2}$. The criterion (22) guarantees that, for all $v \in \mathcal{V}_{W_{i}}$ and for all $p \in \bar{P}$, the output $y$ in the loop of Figure 1 is such that $\|y\|_{2}<\eta\|v\|_{2}$. In other words, the smaller $\eta$, the larger is the rejection rate for the disturbances $v \in \mathcal{V}_{W_{i}}$.

In the case of LPI control, the disturbance rejection ability can be ensured by imposing the same criterion, but now for all scheduling sequences $p(t)$ in $\mathbf{P}$ instead of $\bar{P}$ :

$$
\sup _{p \in \mathbf{P}}\left\|\hat{\bar{S}}(p(t)) W_{i}\right\|_{\mathcal{L}_{2}}=\sup _{\mathbf{p} \in \mathcal{P}}\left\|\frac{W_{i}(z)}{1+\hat{C}(z, \mathbf{p}) G(z, T(\mathbf{p}) \hat{\theta})}\right\|_{\infty}<\eta
$$

with the notations introduced in the previous subsection. The performance require- 
ment (23) can also be equivalently rewritten as:

$$
\sup _{\mathbf{p} \in \mathcal{P}}\left|\frac{1}{1+\hat{C}\left(e^{j \omega}, \mathbf{p}\right) G\left(e^{j \omega}, T(\mathbf{p}) \hat{\theta}\right)}\right|<\frac{\eta}{\left|W_{i}\left(e^{j \omega}\right)\right|} \quad \forall \omega
$$

Finally, note that, in addition to the input weighting $W_{i}$, output weightings can also be added in the performance requirements used in the control design methods $[13,29]$.

\section{Robustness analysis}

\subsection{Concepts}

As shown in the previous section, LPI and LPV control design methods can be used to determine, based on the model $\bar{G}(T(p(t)) \hat{\theta})$, a controller $\hat{\bar{C}}(p(t))$. This controller achieves a certain level of (nominal) performance for a given class $P$ of scheduling sequences ( $P$ is either $\bar{P}$ in the LPV control situation or $\mathbf{P}$ in the LPI control situation). As shown in the previous subsection, when considering the objective of disturbance rejection, this level of performance can be measured as: $J_{\text {nom }}=\max _{p \in P}\left\|\hat{\bar{S}}(p(t)) W_{i}\right\|_{\mathcal{L}_{2}}$. Other performance requirements (i.e. on the control efforts) can also be defined and treated in a similar way as the one on disturbance rejection considered below.

The control design method ensures that $J_{\text {nom }}<\eta$ for an user-chosen $\eta<1$. However, this performance level only pertains to the loop $[\hat{\bar{C}}(p(t)) \bar{G}(T(p(t)) \hat{\theta})]$. In particular, this level of performance is not guaranteed when $\hat{\bar{C}}(p(t))$ is applied to the (unknown) true system $\bar{G}\left(T(p(t)) \theta^{0}\right)$ (since $\left.\hat{\theta} \neq \theta^{0}\right)$. Consider thus the loop $\left[\hat{\bar{C}}(p(t)) \bar{G}\left(T(p(t)) \theta^{0}\right)\right]$ which is the loop in Figure 1 where $\bar{G}(T(p(t)) \hat{\theta})$ is replaced by $\bar{G}\left(T(p(t)) \theta^{0}\right)$. In order to verify the robustness of the designed controller $\hat{\bar{C}}(p(t))$, we have to verify that

$$
J_{0}=\sup _{p \in P}\left\|\bar{S}_{0}(p(t)) W_{i}\right\|_{\mathcal{L}_{2}}<\eta_{r o b}
$$

where $\bar{S}_{0}(p(t))$ is the operator between $v$ and $y$ in this loop $\left[\hat{\bar{C}}(p(t)) \bar{G}\left(T(p(t)) \theta^{0}\right)\right]$ and where $\eta_{\text {rob }}>\eta$ is an user-chosen scalar (the difference $\eta_{\text {rob }}-\eta$ thus represents the maximal performance degradation that can be allowed).

Since $\theta^{0}$ is unknown, in order to verify (25), we will consider the uncertainty ellipsoid $U$ defined in (14) and, more precisely, the following quantity representing the worst case performance with respect to $U$ :

$$
J_{w c}=\sup _{\delta \theta \in U} \sup _{p \in P}\left\|\bar{S}_{\delta \theta}(p(t)) W_{i}\right\|_{\mathcal{L}_{2}}
$$

where $\bar{S}_{\delta \theta}(p(t))$ is the operator between $v$ and $y$ in the loop $[\hat{\bar{C}}(p(t)) \bar{G}(T(p(t)) \theta)]$ made up of the designed controller $\hat{\bar{C}}(p(t))$ and the plant $\bar{G}(T(p(t)) \theta)$ with $\theta=\hat{\theta}+$ $\delta \theta([\hat{\bar{C}}(p(t)) \bar{G}(T(p(t)) \theta)]$ is the loop in Figure 1 where $\bar{G}(T(p(t)) \hat{\theta})$ is replaced by $\bar{G}(T(p(t)) \theta))$.

Like in many robustness analysis problems [36], it will not be possible to compute the quantity $J_{w c}$ exactly. However, using the separation of graph theory $[28,18,25]$, we 


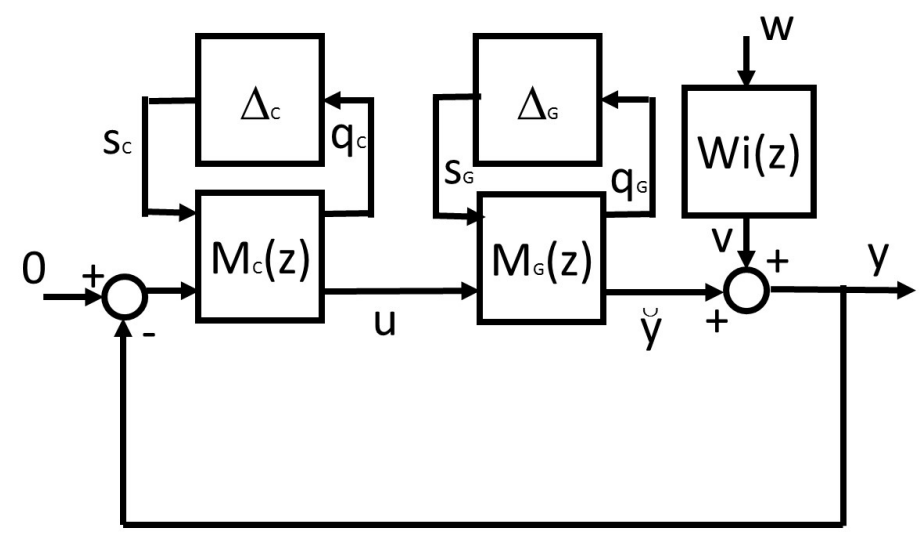

Figure 2.: The closed loop $[\hat{\bar{C}}(p(t)) \bar{G}(T(p(t)) \theta)]$ for an arbitrary $\theta$ using the LFT representations

will see in the sequel that we can compute an upper bound $J_{w c}^{u b}$ for $J_{w c}$ using convex optimization. Based on this computable upper bound for $J_{w c},(25)$ will be deemed verified if

$$
J_{w c}^{u b}<\eta_{\text {rob }}
$$

To find a method to compute $J_{w c}^{u b}$, it is important to note that $\bar{G}(T(p(t)) \theta)$ can be expressed as an LFT in $p$ and in $\delta \theta=\theta-\hat{\theta}$ (see (15)) and to recall that the designed controller $\hat{\bar{C}}(p(t))$ has the property to be rational in $p$ (See Section 3.1), i.e., we can define signal $s_{C}$ and $q_{C}$ such that the operator $\hat{\bar{C}}(p(t))$ can be expressed as the following LFT:

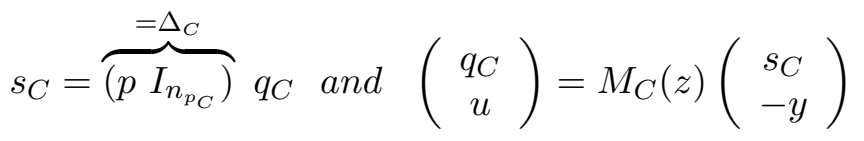

for some given scalar ${ }^{6} n_{p_{C}}$ and some matrix of transfer functions $M_{C}(z)$.

Consequently, the operator $\bar{S}_{\delta \theta}(p(t)) W_{i}$ can also be expressed as an LFT in $p$ and in $\delta \theta$, i.e., for $q=\left(q_{C}^{T}, q_{G}^{T}\right)^{T}$ and $s=\left(s_{C}^{T}, s_{G}^{T}\right)^{T}$, the operator $\bar{S}_{\delta \theta}(p(t)) W_{i}$ between $w$ and $y$ (see Figure 2) can be written as:

$$
s=\overbrace{\operatorname{bdiag}\left(p I_{n_{p}}, I_{n_{\theta}} \otimes \delta \theta\right)}^{=\Delta} q \text { and }\left(\begin{array}{c}
q \\
y
\end{array}\right)=M(z)\left(\begin{array}{c}
s \\
w
\end{array}\right)
$$

with $\Delta=\operatorname{bdiag}\left(\Delta_{C}, \Delta_{G}\right)\left(n_{p}=n_{p_{C}}+n_{p_{G}}\right.$ and $\left.n_{\theta}=n_{\theta_{G}}\right)$ and with $M(z)$ a known matrix of stable LTI transfer functions. These transfer functions are stable since the loop $[\hat{\bar{C}}(p(t)=0) \bar{G}(T(p(t)=0) \hat{\theta})]$ corresponding to $\delta \theta=0$ and $p(t)=0$ is stable (see

\footnotetext{
${ }^{6}$ If the control design methods in, e.g., $[13,29]$ are used, we have that $n_{p_{C}}=n_{p_{G}}$.
} 
Section 3.1). In the sequel, we will use the shorthand relation $\mathcal{F}(.,$.$) for such a LFT$ representation (29), i.e., $\bar{S}_{\delta \theta}(p(t)) W_{i}=\mathcal{F}(M(z), \Delta)$.

Remark 1. The operator $\bar{S}_{\delta \theta}(p(t))$ (without weighting $W_{i}$ ) can of course also be expressed as an LFT using the same uncertainty block $\Delta$, i.e., $\bar{S}_{\delta \theta}(p(t))=\mathcal{F}(\tilde{M}(z), \Delta)$ where $\tilde{M}(z)$ is also a known matrix of stable LTI transfer functions.

In the next subsections, we will see how $J_{w c}^{u b}$ can be computed in practice.

\subsection{Separation of graph}

Let us first notice that the worst-case performance $J_{w c}$ defined in (26) is the square root $\sqrt{\gamma_{o p t}}$ of the solution $\gamma_{\text {opt }}$ of the following optimization problem:

$$
\begin{gathered}
\min _{\gamma} \gamma \\
\|\mathcal{F}(M(z), \Delta)\|_{\mathcal{L}_{2}}^{2}<\gamma \quad \forall \Delta \in \boldsymbol{\Delta}
\end{gathered}
$$

where $\boldsymbol{\Delta}$ is either $\boldsymbol{\Delta}_{\bar{P}}$ in the LPV case or $\boldsymbol{\Delta}_{\mathbf{P}}$ in the LPI case:

$$
\boldsymbol{\Delta}_{\bar{P}}=\left\{\Delta=\operatorname{bdiag}\left(p I_{n_{p}}, I_{n_{\theta}} \otimes \delta \theta\right) \mid p \in \bar{P} \text { and } \delta \theta \in U\right\}
$$

$$
\boldsymbol{\Delta}_{\mathbf{P}}=\left\{\Delta=\operatorname{bdiag}\left(p I_{n_{p}}, I_{n_{\theta}} \otimes \delta \theta\right) \mid p \in \mathbf{P} \text { and } \delta \theta \in U\right\}
$$

Remark 2. The set $\boldsymbol{\Delta}_{\mathbf{P}}$ can also be rewritten as:

$$
\boldsymbol{\Delta}_{\mathbf{P}}=\left\{\Delta=\operatorname{bdiag}\left(\mathbf{p} I_{n_{p}}, I_{n_{\theta}} \otimes \delta \theta\right) \mid \mathbf{p} \in \mathcal{P} \text { and } \delta \theta \in U\right\}
$$

and can consequently be considered as a fully time-invariant uncertainty set.

As already mentioned before, the optimization problem (30) yielding $J_{w c}$ is not tractable. However, we will show in this section that if a certain LMI constraint is satisfied, then the constraint in (30) is also satisfied. Replacing the non-tractable constraint in (30) by this LMI constraint yields a convex optimization problem delivering the upper-bound $J_{w c}^{u b}$ on $J_{w c}$. This LMI constraint will be given in Proposition 1. A necessary ingredient for this proposition is to associate, with the set $\boldsymbol{\Delta}$ (i.e. $\boldsymbol{\Delta}_{\bar{P}}$ for the LPV situation or $\boldsymbol{\Delta}_{\mathbf{P}}$ in the LPI situation), a so-called set of multipliers. In a nutshell, this set of multipliers is an explicit parametrization of the quadratic constraints satisfied by the graphs of all uncertainties $\Delta \in \boldsymbol{\Delta}[28,18,25]$. Since $p$ may be time-varying, we cannot define these quadratic constraints on a frequency-wise manner (like in, e.g., [5]), but we will instead use Integral Quadratic Constraints (IQC) [25].

Definition 2. Consider a set $\boldsymbol{\Delta}$ of the form (31) or of the form (32). Consider also the set $\mathcal{G}_{\boldsymbol{\Delta}}$ containing all $(q(t) s(t))^{T}$ that can be generated by an element $\Delta$ in such 
a set $\boldsymbol{\Delta}$ :

$$
\mathcal{G}_{\boldsymbol{\Delta}}=\left\{\left(\begin{array}{c}
q \\
s
\end{array}\right) \in \mathcal{L}_{2} \mid s(t)=\Delta q(t) \text { with } q \in \mathcal{L}_{2} \text { and } \Delta \in \boldsymbol{\Delta}\right\} .
$$

The set $\mathcal{A}$ of multipliers associated to $\Delta$ is a set of affinely parametrized and frequencydependent Hermitian matrices $A(\omega)$ and all frequency-dependent matrices $A(\omega) \in \mathcal{A}$ satisfy the following Integral Quadratic Constraint (IQC) for all $(q(t) s(t))^{T} \in \mathcal{G}_{\Delta}$ :

$$
\frac{1}{2 \pi} \int_{-\pi}^{\pi}\left(\begin{array}{c}
q\left(e^{j \omega}\right) \\
s\left(e^{j \omega}\right)
\end{array}\right)^{*} \underbrace{\left(\begin{array}{cc}
A_{11}(\omega) & A_{12}(\omega) \\
A_{12}^{*}(\omega) & A_{22}(\omega)
\end{array}\right)}_{=A(\omega)}\left(\begin{array}{c}
q\left(e^{j \omega}\right) \\
s\left(e^{j \omega}\right)
\end{array}\right) d \omega \geq 0
$$

with $q\left(e^{j \omega}\right)$ and $s\left(e^{j \omega}\right)$ the Fourier transforms of $q(t)$ and $s(t)$.

We will in the sequel determine a specific set of multipliers $\mathcal{A}_{\bar{P}}$ for the uncertainty set $\boldsymbol{\Delta}_{\bar{P}}$ corresponding to the LPV situation and one specific set $\mathcal{A}_{\mathbf{P}}$ for the uncertainty set $\boldsymbol{\Delta}_{\mathbf{P}}$ corresponding to the LPI situation. However, let us first show how this set of multipliers can be used to obtain a tractable condition that implies $\|\mathcal{F}(M(z), \Delta)\|_{\mathcal{L}_{2}}<\gamma \quad \forall \Delta \in \boldsymbol{\Delta}$ (i.e. the constraint in (30)).

Proposition 1. Consider an LFT representation $\mathcal{F}(M(z), \Delta)$ as given in (29) where $M(z)$ is a stable matrix of LTI transfer functions and $\Delta$ is a stable operator lying in the set $\boldsymbol{\Delta}$ (given by (31) for the LPV situation or by (32) in the LPI situation). Suppose that we have determined a set $\mathcal{A}$ of multipliers associated to $\boldsymbol{\Delta}$ (see Definition 2). Then, if there exist a frequency-dependent matrix $A(\omega) \in \mathcal{A}$ and a positive scalar $\gamma<\infty$ such that ${ }^{7}$ :

$$
\begin{gathered}
\left(\begin{array}{c}
M\left(e^{j \omega}\right) \\
I
\end{array}\right)^{*} \mathcal{B}(A(\omega), \gamma)\left(\begin{array}{cc}
M\left(e^{j \omega}\right) \\
I
\end{array}\right)<0 \quad \forall \omega \\
\mathcal{B}(A(\omega), \gamma)=\left(\begin{array}{cc}
\operatorname{bdiag}\left(A_{11}(\omega), 1\right) & \operatorname{bdiag}\left(A_{12}(\omega), 0\right) \\
\operatorname{bdiag}\left(A_{12}^{*}(\omega), 0\right) & \operatorname{bdiag}\left(A_{22}(\omega),-\gamma\right)
\end{array}\right),
\end{gathered}
$$

then this implies both the stability of $\mathcal{F}(M(z), \Delta)$ for all $\Delta \in \boldsymbol{\Delta}$ and the following property:

$$
\|\mathcal{F}(M(z), \Delta)\|_{\mathcal{L}_{2}}^{2}<\gamma \quad \forall \Delta \in \Delta
$$

Proof. See Appendix B.

\subsection{Sets of multipliers for $\Delta_{\bar{P}}$ and $\Delta_{\mathrm{P}}$}

To be able to use Proposition 1 in order to compute $J_{w c}^{u b}$ in the LPV and LPI situations, we need to associate a set of multipliers to both $\boldsymbol{\Delta}_{\bar{P}}$ and $\boldsymbol{\Delta}_{\mathbf{P}}$. This is done in the next two propositions. It is important to stress that the more extensive the parametrization of the set of multipliers, the closer the upper bound $J_{w c}^{u b}$ will

${ }^{7} \operatorname{In}(35), I$ is the identity matrix with dimension $n_{p}+k n_{\theta}+1$. 
be from $J_{w c}$. With respect to the classical literature on LPV robustness analysis $[25,26,35,20,6,1]$, the main difference is the second block-diagonal term $I_{n_{\theta}} \otimes \delta \theta$ in the uncertainty $\Delta$ in (29), i.e., the uncertainty related to the estimation error following from the LPV identification procedure. Such an uncertainty block has been treated in [5] in the case where the identified system was an LTI transfer function (i.e. without scheduling variable $p$ ). With respect to [5], the difference is thus the combination of this uncertainty block $I_{n_{\theta}} \otimes \delta \theta$ with the uncertainty block made up of a repeated scalar $p$ which is either time-varying or time-invariant.

Proposition 2. Consider the set $\boldsymbol{\Delta}_{\bar{P}}$ defined in (31) where $\bar{P}$ is defined in (19) and $U$ is defined in (14). The frequency-dependent matrices $A(\omega)=\left(\begin{array}{cc}A_{11}(\omega) & A_{12}(\omega) \\ A_{12}^{*}(\omega) & A_{22}(\omega)\end{array}\right)$ belonging to the set $\mathcal{A}_{\bar{P}}$ of multipliers associated to $\boldsymbol{\Delta}_{\bar{P}}$ are of the form $A_{i j}(\omega)=$ $\operatorname{bdiag}\left(A_{i j, p}(\omega), A_{i j, \delta \theta}(\omega)\right) \quad(i, j=1,2)$.

The matrices $A_{i j, p}(\omega)$ are square matrices of dimension $n_{p}$ that are parametrized as follows: $A_{11, p}(\omega)=p_{\max }^{2} \mathcal{S}, A_{12, p}(\omega)=\mathcal{G}$ and $A_{22, p}(\omega)=-\mathcal{S}$ where $p_{\max }$ is defined in (1) and where $\mathcal{S}$ and $\mathcal{G}$ can take any value provided that i) $\mathcal{S}$ is a positive definite Hermitian matrix, ii) $\mathcal{G}$ is a skew-symmetric matrix and iii) $\mathcal{S}$ and $\mathcal{G}$ do not depend on the frequency $\omega$.

At any frequency $\omega$, the matrices $A_{i j, \delta \theta}(\omega)$ are parametrized as follows:

$$
\begin{gathered}
A_{11, \delta \theta}(\omega)=A_{0}(\omega) \\
A_{12, \delta \theta}(\omega)=\left(\begin{array}{cccc}
j \hat{v}_{11}^{T}(\omega) & j \hat{v}_{12}^{T}(\omega) & \ldots & j \hat{v}_{1 n_{\theta}}^{T}(\omega) \\
j \hat{v}_{12}^{T}(\omega) & j \hat{v}_{22}^{T}(\omega) & \ldots & j \hat{v}_{2 n_{\theta}}^{T}(\omega) \\
\vdots & & \ddots & \vdots \\
j \hat{v}_{1 n_{\theta}}^{T}(\omega) & \ldots & \ldots & j \hat{v}_{n_{\theta} n_{\theta}}^{T}(\omega)
\end{array}\right)+\ldots \\
\\
\ldots+\left(\begin{array}{ccccc}
0 & \tilde{v}_{12}^{T}(\omega) & \ldots & \\
-\tilde{v}_{12}^{T}(\omega) & 0 & \ldots & \tilde{v}_{1 n_{\theta}}^{T}(\omega) \\
\vdots & \ldots & \ddots & \vdots \\
-\tilde{v}_{1 n_{\theta}}^{T}(\omega) & \ldots & -\tilde{v}_{\left(n_{\theta}-1\right) n_{\theta}}^{T}(\omega) & \tilde{v}_{\left(n_{\theta}-1\right) n_{\theta}}^{T}(\omega) \\
\end{array}\right.
\end{gathered}
$$

where $A_{0}(\omega)$ is a square matrix of dimension $n_{\theta}$ that can take any value as long as $A_{0}(\omega)$ is a positive definite Hermitian matrix, where $\hat{v}_{l r}(\omega)$ and $\tilde{v}_{l r}(\omega)$ are column vectors of dimension $k$ that can take any value $\left(l=1, . ., n_{\theta}\right.$ and $\left.r=1, . ., n_{\theta}\right)$ and finally where $\tilde{A}(\omega)$ and $\tilde{B}(\omega)$ are square matrix of dimension $k n_{\theta}$ that can take any values as long as:

$$
\begin{gathered}
\tilde{A}=\left(\begin{array}{cccc}
L_{11}(\omega) & L_{12}(\omega) & \ldots & L_{1 n_{\theta}}(\omega) \\
L_{12}(\omega) & L_{22}(\omega) & \ldots & L_{2 n_{\theta}}(\omega) \\
\vdots & & \ddots & \vdots \\
L_{1 n_{\theta}}(\omega) & L_{2 n_{\theta}}(\omega) & \ldots & L_{n_{\theta} n_{\theta}}(\omega)
\end{array}\right) \\
\tilde{B}=\left(\begin{array}{cccc}
0 & K_{12}(\omega) & \ldots & K_{1 n_{\theta}}(\omega) \\
-K_{12}(\omega) & 0 & \ldots & \vdots \\
\vdots & & \ddots & K_{\left(n_{\theta}-1\right) n_{\theta}}(\omega) \\
-K_{1 n_{\theta}}(\omega) & \ldots & -K_{\left(n_{\theta}-1\right) n_{\theta}}(\omega) & 0
\end{array}\right)
\end{gathered}
$$


with the constraints that $L_{i, j}(\omega)=L_{i, j}(\omega)^{T} \in \mathbf{R}^{k \times k}$ and $K_{i, j}(\omega)=-K_{i, j}^{T}(\omega) \in \mathbf{R}^{k \times k}$. Finally, as long as they keep the structure defined above, the elements $A_{0}(\omega), \tilde{A}(\omega)$, $\tilde{B}(\omega), \hat{v}_{l r}(\omega)$ and $\tilde{v}_{l r}(\omega)$ can take different values at each frequency $\omega$.

Proof. See Appendix C.

Proposition 3. Consider the set $\boldsymbol{\Delta}_{\mathbf{P}}$ defined in (33) where $\mathcal{P}$ is defined in (1) and $U$ is defined in (14). The frequency-dependent matrices $A(\omega)=\left(\begin{array}{cc}A_{11}(\omega) & A_{12}(\omega) \\ A_{12}^{*}(\omega) & A_{22}(\omega)\end{array}\right)$ belonging to the set $\mathcal{A}_{\mathbf{P}}$ of multipliers associated to $\boldsymbol{\Delta}_{\mathbf{P}}$ are of the form $A_{i j}(\omega)=$ $\operatorname{bdiag}\left(A_{i j, p}(\omega), A_{i j, \delta \theta}(\omega)\right)(i, j=1,2)$.

At any frequency $\omega$, the matrices $A_{i j, p}(\omega)$ are square matrices of dimension $n_{p}$ that are parametrized as follows: $A_{11, p}(\omega)=p_{\max }^{2} \mathcal{S}(\omega), A_{12, p}(\omega)=\mathcal{G}(\omega)$ and $A_{22, p}(\omega)=-\mathcal{S}(\omega)$ where $p_{\max }$ is defined in (1) and where $\mathcal{S}(\omega)$ and $\mathcal{G}(\omega)$ can take any value provided that i) $\mathcal{S}(\omega)$ is a positive definite Hermitian matrix, ii) $\mathcal{G}(\omega)$ is a skew-symmetric matrix. Moreover, as long as they keep the structure defined above, the matrices $\mathcal{S}(\omega)$ and $\mathcal{G}(\omega)$ can take different values at each frequency $\omega$.

Finally, the matrices $A_{i j, \delta \theta}(\omega)$ are parametrized in $\mathcal{A}_{\mathbf{P}}$ in the same way as in $\mathcal{A}_{\bar{P}}$ (see Proposition 2).

\section{Proof. See Appendix D.}

The parametrization of the frequency-dependent matrices $A(\omega)$ in Propositions 2 and 3 is made up of fixed elements, i.e., $p_{\max }$ and $P_{\theta}^{-1} / \chi$ and of free variables that appear affinely in the parametrization. In the case of $\mathcal{A}_{\bar{P}}$, these free variables are $\mathcal{S}, \mathcal{G}$, $A_{0}(\omega), \tilde{A}(\omega), \tilde{B}(\omega), \hat{v}_{l r}(\omega)$ and $\tilde{v}_{l r}(\omega)\left(l=1, . ., n_{\theta}\right.$ and $\left.r=1, . ., n_{\theta}\right)$. In the case of $\mathcal{A}_{\mathbf{P}}$, these free variables are $\mathcal{S}(\omega), \mathcal{G}(\omega), A_{0}(\omega), \tilde{A}(\omega), \tilde{B}(\omega), \hat{v}_{l r}(\omega)$ and $\tilde{v}_{l r}(\omega)\left(l=1, . ., n_{\theta}\right.$ and $\left.r=1, . ., n_{\theta}\right)$. When, such as in Proposition 1, we have to find $A(\omega)$ in a set of multipliers, it is in fact those free variables that have to be determined (according to the defined structure for each of these variables). In the case of $\mathcal{A}_{\bar{P}}$, some of these free variables (i.e. $\mathcal{S}$ and $\mathcal{G}$ ) are constant over the frequencies while, in the case of $\mathcal{A}_{\mathbf{P}}$, the values of all these free variables can be determined independently and differently at each frequency $\omega$. This last property is a consequence of the fact that $\boldsymbol{\Delta}_{\mathbf{P}}$ is in fact a time-invariant uncertainty set (see Remark 2) and the set $\mathcal{A}_{\mathbf{P}}$ of multipliers associated to $\boldsymbol{\Delta}_{\mathbf{P}}$ deduced from Definition 2 corresponds to the set that would have been deduced from the (frequency-wise) definition of the set of multipliers for timeinvariant uncertainty sets (this definition is, e.g., used in our previous contribution $[5])$.

\subsection{Computation of the upper bound for $J_{w c}$}

Based on the discussion in the previous subsections, an upper bound $J_{w c}^{u b}$ for the worst-case performance $J_{w c}$ defined in (26) (see also (30)) can be derived using a convex LMI optimization problem [11] both for the LPI and LPV situations. This is summarized in the following proposition. Using the quantity $J_{w c}^{u b}$ computed with this convex optimization problem, we will be able to verify the robustness condition (27) and, consequently, whether the controller $\hat{\bar{C}}(p(t))$ designed based on the identified model $\bar{G}(T(p(t)) \hat{\theta})$ will achieve sufficient performance with the unknown true system 
$\bar{G}\left(T(p(t)) \theta^{0}\right)$ for all scheduling sequences $p \in \mathbf{P}$ (in the LPI situation) or $p \in \bar{P}$ (in the LPV situation).

Proposition 4. Consider the worst case performance $J_{w c}$ defined as shown in Section 4.2 via the solution of the optimization problem (30) with $\boldsymbol{\Delta}=\boldsymbol{\Delta}_{\bar{P}}$ in the $L P V$ situation and with $\boldsymbol{\Delta}=\boldsymbol{\Delta}_{\mathbf{P}}$ in the LPI situation (see (31)-(32)). Consider also the sets $\mathcal{A}_{\bar{P}}$ and $\mathcal{A}_{\mathbf{P}}$ of multipliers associated respectively to $\boldsymbol{\Delta}_{\bar{P}}$ and $\boldsymbol{\Delta}_{\mathbf{P}}$ (see Propositions 2 and 3). An upper bound $J_{w c}^{u b}$ for $J_{w c}$ can be derived using convex LMI optimization in both the LPV situation and the LPI situation. Indeed, in the LPV situation, $J_{w c}^{u b}$ is the square root of the smallest value of $\gamma$ for which we can still find a frequency-dependent matrix $A(\omega) \in \mathcal{A}_{\bar{P}}$ such that (35) holds for these $A(\omega)$ and $\gamma$. Similarly, in the LPI situation, $J_{w c}^{u b}$ is the square root of the smallest value of $\gamma$ for which we can still find a frequency-dependent matrix $A(\omega) \in \mathcal{A}_{\mathbf{P}}$ such that (35) holds for these $A(\omega)$ and $\gamma$.

Proof. That $J_{w c}^{u b}$ is an upper bound for $J_{w c}$ is a direct consequence of Proposition 1. That the optimization problems yielding $J_{w c}^{u b}$ is a convex LMI optimization problem is a consequence of the fact that (35) is affine in both $A(\omega)$ and $\gamma$ and that the tobe-determined free variables appear in an affine manner in the parametrization of the matrices $A(\omega)$ given in Propositions 2 and 3.

The constraints in both optimization problems presented in Proposition 4 are given by (35) which specify an LMI constraint for all frequencies $\omega \in[-\pi, \pi]$. The LMI optimization problem has thus an infinite number of constraints. A possible solution to this difficulty is to factorize the matrices $\mathcal{B}(A(\omega), \gamma)$ as $\mathcal{L}^{*}\left(e^{j \omega}\right) \Pi \mathcal{L}\left(e^{j \omega}\right)$ with $\mathcal{L}(z)$ a matrix containing known basis functions and $\Pi$ a matrix containing free coefficients ${ }^{8}$. This factorization would allow to use the KYP lemma [11] to replace the infinite number of LMI constraints in (35) by one single LMI constraint. Another solution is to replace the infinite number of constraints (i.e. one for each $\omega$ in the continuous interval $[-\pi, \pi]$ ) by a finite number of constraints, i.e., one for each frequency in a finite grid $\Omega$ of the interval ${ }^{9}[0, \pi]$ :

$$
\left(\begin{array}{c}
M\left(e^{j \omega}\right) \\
I
\end{array}\right)^{*} \mathcal{B}(A(\omega), \gamma)\left(\begin{array}{c}
M\left(e^{j \omega}\right) \\
I
\end{array}\right)<0 \quad \forall \omega \in \Omega
$$

with $\mathcal{B}(.,$.$) defined in the same way as in (35). If we use (37) instead of (35) in the$ optimization problems of Proposition 4, the determination of a frequency-dependent matrix $A(\omega)$ is replaced by the determination of the value of $A(\omega)$ at each frequency $\omega$ in the finite grid $\Omega$. Even though it possibly entails some approximation, this gridding approach will be the approach considered in this paper for its simplicity.

\footnotetext{
${ }^{8}$ To find an appropriate factorization for $\mathcal{B}$, the approach presented in Proposition 1 of [8] can, e.g., be followed.

${ }^{9}$ Note that we here use the symmetry between positive and negative frequencies.
} 


\subsection{Specific robustness analysis approach in the LPI situation}

\subsubsection{Approach based on graph separation}

Due to the fact that the value of the frequency-dependent matrix $A(\omega)$ can be chosen independently at each frequency in the LPI situation (see Proposition 3), another approach (which is even simpler) can also be considered for this case, i.e., solving, for each frequency $\omega$ in the finite grid $\Omega$ of the interval $[0 \pi]$, an LMI optimization problem with one single LMI constraint. The LMI optimization problem considered at one given frequency $\omega$ in this grid is the following one: determine the smallest value of the scalar $\gamma(\omega)$ for which we can still find a matrix $A(\omega)$ having the structure given in Proposition 3 such that, for these $A(\omega)$ and $\gamma(\omega)$, the following LMI constraint holds:

$$
\left(\begin{array}{c}
M\left(e^{j \omega}\right) \\
I
\end{array}\right)^{*} \mathcal{B}(A(\omega), \gamma(\omega))\left(\begin{array}{c}
M\left(e^{j \omega}\right) \\
I
\end{array}\right)<0
$$

When we speak here of a matrix $A(\omega)$ having the structure given in Proposition 3, we mean a matrix $A(\omega)$ which is equal to the value at $\omega$ of a frequency-dependent matrix in $\mathcal{A}_{\mathbf{P}}$.

If we denote by $J_{w c}^{u b}(\omega)$ the square root of the solution $\gamma_{o p t}(\omega)$ of this optimization problem at a given frequency $\omega$, we have the following result.

Proposition 5. Consider an $L F T$ representation $\mathcal{F}(M(z), \Delta)$ with $M(z)$ a stable matrix of transfer functions and $\Delta$ a (time-invariant) operator that lies in the uncertainty set $\boldsymbol{\Delta}_{\mathbf{P}}$ (see (33)). Consider also the optimization problem defined above yielding a solution $J_{w c}^{u b}(\omega)$ at a given $\omega$. Then, at any $\omega$, we have that $J_{w c}^{u b}(\omega)$ is an upper bound for:

$$
J_{w c}(\omega)=\max _{\Delta \in \Delta_{\mathbf{P}}}\left|\mathcal{F}\left(M\left(e^{j \omega}\right), \Delta\right)\right|
$$

and the upper bound $J_{w c}^{u b}$ defined in Proposition 4 for the LPI case is equal to $\sup _{\omega} J_{w c}^{u b}(\omega)$.

Proof. See Appendix E.

As already mentioned, it is clear from Proposition 5 that the robustness condition (27) in the LPI case can be checked by computing $J_{w c}^{u b}(\omega)$ at all frequencies in a fine grid $\Omega$ of the interval $[0 \pi]$ and by verifying that $J_{w c}^{u b}(\omega)<\eta_{r o b}$ at all these frequencies. This also means that the LMI optimization problem with multiple LMI constraints (37) will in practice only be used for the LPV case.

Remark 3. Note that the expression (39) valid for the LPI case is equivalent to:

$$
J_{w c}(\omega)=\sup _{\delta \theta \in U} \sup _{\mathbf{p} \in \mathcal{P}}\left|\frac{W_{i}\left(e^{j \omega}\right)}{1+\hat{C}\left(e^{j \omega}, \mathbf{p}\right) G\left(e^{j \omega}, T(\mathbf{p})(\hat{\theta}+\delta \theta)\right)}\right|
$$


Using Proposition 5 and Remark 1, one can also compute an upper bound $\tilde{J}_{w c}^{u b}(\omega)$ for

$$
\tilde{J}_{w c}(\omega)=\sup _{\delta \theta \in U} \sup _{\mathbf{p} \in \mathcal{P}}\left|\frac{1}{1+\hat{C}\left(e^{j \omega}, \mathbf{p}\right) G\left(e^{j \omega}, T(\mathbf{p})(\hat{\theta}+\delta \theta)\right)}\right|
$$

In this case, verifying $(27)$ consists in checking that $\tilde{J}_{w c}^{u b}(\omega)<\eta_{r o b} /\left|W_{i}\left(e^{j \omega}\right)\right|$ at all frequencies in a fine grid $\Omega$ of $[0, \pi]$.

\subsubsection{Approach based on the gridding of $\mathcal{P}$}

The approach presented in the previous subsection allows one to compute an upper bound $J_{w c}^{u b}(\omega)$ for the quantity $J_{w c}(\omega)$ defined in, e.g., (40). In the sequel, we will show that we can easily also compute a reliable lower bound $J_{w c}^{l b}(\omega)$ of $J_{w c}(\omega)$ by using the fact that the scheduling space is of dimension one (and can thus be easily gridded). A lower bound could seem less attractive than an upper bound. Indeed, verifying that $J_{w c}^{l b}(\omega)<\eta_{r o b}$ is no guarantee for $J_{w c}(\omega)<\eta_{\text {rob }}$ (unlike $J_{w c}^{u b}(\omega)<\eta_{\text {rob }}$ ). However, we will see in the next section that this lower bound can lead to an interesting alternative/initialization for the optimal experiment design problem. Moreover, this lower bound can be used as a tool to verify the conservatism of $J_{w c}^{u b}(\omega)$ : this conservatism is limited if $J_{w c}^{l b}(\omega) \approx J_{w c}^{u b}(\omega) \forall \omega$.

Let us see how we can determine a reliable lower bound $J_{w c}^{l b}(\omega)$ for $J_{w c}(\omega)$. For this purpose, observe that $J_{w c}(\omega)$ can be rewritten as

$$
\begin{gathered}
J_{w c}(\omega)=\sup _{\mathbf{p} \in \mathcal{P}} J_{w c}(\omega, \mathbf{p}) \\
\text { with } J_{w c}(\omega, \mathbf{p}) \triangleq \sup _{\delta \theta \in U}\left|\frac{W_{i}\left(e^{j \omega}\right)}{1+\hat{C}\left(e^{j \omega}, \mathbf{p}\right) G\left(e^{j \omega}, T(\mathbf{p})(\hat{\theta}+\delta \theta)\right)}\right|
\end{gathered}
$$

Using our previous results (see, e.g., [7] and Proposition 6 in the sequel), we know that $J_{w c}(\omega, \mathbf{p})$ can be exactly computed for given $\mathbf{p}$ and $\omega$. We can therefore deduce a lower bound $J_{w c}^{l b}(\omega)$ for $J_{w c}(\omega)$ by considering a fine grid $\mathcal{P}^{\text {grid }}$ of the scheduling space $\mathcal{P}:$

$$
J_{w c}^{l b}(\omega)=\sup _{\mathbf{p} \in \mathcal{P}^{g r i d}} J_{w c}(\omega, \mathbf{p})
$$

It is important to note that this lower bound is indeed a reliable lower bound, i.e., the difference between $J_{w c}(\omega)$ and $J_{w c}^{l b}(\omega)$ can be made as small as possible by ever refining $\mathcal{P}^{\text {grid }}$. Let us finish this subsection by recalling how $J_{w c}(\omega, \mathbf{p})$ defined above can indeed be computed exactly for given $\mathbf{p}$ and $\omega$ using convex optimization.

Proposition 6. Consider the quantity $J_{w c}(\omega, \mathbf{p})$ defined in (43) for a given $\omega$, a given $\mathbf{p}$ and a given uncertainty $U$ (see (14)). Consider also the parametrization (16) for $G\left(e^{j \omega}, T(\mathbf{p}) \theta\right)(\theta=\hat{\theta}+\delta \theta)$. Then, $J_{w c}(\omega, \mathbf{p})$ is given by $\sqrt{\gamma_{o p t}}$ where $\gamma_{o p t}$ is the solution of the following LMI convex optimization problem: determine the smallest value of $\gamma$ 
for which we can still determine a positive scalar $\tau$ such that, for these $\gamma$ and $\tau$, the following LMI holds:

$$
\begin{gathered}
\mathcal{E}(\omega, \mathbf{p})-\gamma \mathcal{E}_{\gamma}(\omega, \mathbf{p})-\tau\left(\begin{array}{cc}
P_{\theta}^{-1} & P_{\theta}^{-1} \hat{\theta} \\
P_{\theta}^{-1} \hat{\theta} & \chi-\hat{\theta}^{T} P_{\theta}^{-1} \hat{\theta}
\end{array}\right)<0 \\
\mathcal{E}(\omega, \mathbf{p})=\left|W_{i}\left(e^{j \omega}\right)\right|^{2}\left(\begin{array}{cc}
\operatorname{Re}\left(Z_{D}^{*}\left(e^{j \omega}, \mathbf{p}\right) Z_{D}\left(e^{j \omega}, \mathbf{p}\right)\right) & \operatorname{Re}\left(Z_{D}^{*}\left(e^{j \omega}, \mathbf{p}\right)\right) \\
\operatorname{Re}\left(Z_{D}\left(e^{j \omega}, \mathbf{p}\right)\right) & 1
\end{array}\right) \\
\mathcal{E}_{\gamma}(\omega, \mathbf{p})=\left(\begin{array}{cc}
\operatorname{Re}\left(Z_{1}^{*}\left(e^{j \omega}, \mathbf{p}\right) Z_{1}\left(e^{j \omega}, \mathbf{p}\right)\right) & \operatorname{Re}\left(Z_{1}^{*}\left(e^{j \omega}, \mathbf{p}\right)\right) \\
\operatorname{Re}\left(Z_{1}\left(e^{j \omega}, \mathbf{p}\right)\right) & 1
\end{array}\right)
\end{gathered}
$$

where $\operatorname{Re}(a)$ is the real part of the complex quantity a and $Z_{1}\left(e^{j \omega}, \mathbf{p}\right) \triangleq$ $\hat{C}\left(e^{j \omega}, \mathbf{p}\right) Z_{N}\left(e^{j \omega}, \mathbf{p}\right)+Z_{D}\left(e^{j \omega}, \mathbf{p}\right)$. Note that the optimal $\gamma$ and $\tau$ can be different for different $\omega$ and $\mathbf{p}$.

Proof. As shown in [7], the proposition follows from the fact that the existence of $\tau>0$ such that (45) holds is equivalent to:

$$
\left|\frac{W_{i}\left(e^{j \omega}\right)}{1+\hat{C}\left(e^{j \omega}, \mathbf{p}\right) G\left(e^{j \omega}, T(\mathbf{p})(\hat{\theta}+\delta \theta)\right)}\right|^{2}<\gamma \quad \forall \delta \theta \in U
$$

Note that we can similarly also compute a lower bound $\tilde{J}_{w c}^{l b}(\omega)$ for the quantity $\tilde{J}_{w c}(\omega)$ defined in (41) using the procedure described in this section.

Note also that, as mentioned at the end of Section 3.1, the robustness analysis presented in this subsection for the LPI case can also be applied to assess the robustness of an LPV control loop with slowly varying scheduling variable $p$. The performance difference between the LPI case and this slowly varying case will indeed be generally neglectable.

\section{Optimal identification experiment design for LPI/LPV control}

\subsection{Concepts}

In both the LPI and LPV cases, the robustness of the controller designed based on the identified model can be verified by considering the upper bound $J_{w c}^{u b}$ for the worst case performance achieved by this controller over the set of plants that are defined based on the uncertainty set $U$ (see (14)). In some cases, the uncertainty set $U$ will be small enough to guarantee $J_{w c}^{u b}<\eta_{r o b}$. However, in some cases too, the uncertainty set (or, equivalently, the covariance matrix $P_{\theta}$ of $\hat{\theta}$ ) will be too large to guarantee $J_{w c}^{u b}<\eta_{r o b}$. In this section, we will give tools that, in the latter cases, will help designing a second 
LPV identification experiment yielding a new model whose uncertainty will be small enough to guarantee $J_{w c}^{u b}<\eta_{r o b}$ with the controller designed with this new model (using the same (LPI or LPV) control design method as for the initial controller).

For this purpose, let us describe a bit more both the initial and second LPV identification experiments. Without loss of generality, we will suppose that the initial LPV identification experiment (see Section 2.2) has been performed with $M_{1}$ local LTI experiments. This initial identification experiment has delivered an estimate $\hat{\theta}_{1}$ having a covariance matrix $P_{\theta_{1}}$. We thus suppose that, for the controller $\hat{\bar{C}}_{1}(p(t))$ designed based on $\bar{G}\left(T\left(p(t) \hat{\theta}_{1}\right)\right.$, the quantity $J_{w c}^{u b}$ computed with the uncertainty set $U_{1}$ defined with $P_{\theta_{1}}$ is larger than $\eta_{r o b}$. To improve the accuracy of the LPV model, we will design (in an optimal way) a second LPV identification procedure made up of $M$ local LTI experiments (of duration $N_{p}$ each). These $M$ local LTI experiments are performed at the operating points in $\mathbf{P}^{\mathbf{M}} \triangleq\left\{\mathbf{p}_{\mathbf{1}}, \ldots, \mathbf{p}_{\mathbf{M}}\right\}$. Let us also introduce $\Phi\left(\mathbf{P}^{\mathbf{M}}\right) \triangleq\left\{\Phi_{u_{1}}, \ldots, \Phi_{u_{M}}\right\}$ as the set containing the input spectra used in these $M$ local LTI identification experiments.

As also proposed in [16], after the second LPV identification experiment, the information brought during the initial and the second LPV identification procedure will be combined to deliver an estimate $\hat{\theta}$ of $\theta^{0}$ and its covariance matrix $P_{\theta}$. The estimate $\hat{\theta}$ can be deduced as in (12), but with a summation pertaining to the $M_{1}+M$ local experiments performed in both the initial and the second LPV identification procedures. As shown in [16], the covariance matrix $P_{\theta}$ of $\hat{\theta}$ is then given by:

$$
P_{\theta}=\left(P_{\theta_{1}}^{-1}+\sum_{m=1}^{M} T^{T}\left(\mathbf{p}_{\mathbf{m}}\right) P_{\zeta, m}^{-1} T\left(\mathbf{p}_{\mathbf{m}}\right)\right)^{-1}
$$

where $\mathbf{p}_{\mathbf{m}}(m=1, \ldots, M)$ are the $M$ operating points in $\mathbf{P}^{\mathbf{M}}$ and where $P_{\zeta, m}^{-1}$ is defined in (11) as a function of the spectrum $\Phi_{u_{m}}(\omega)(m=1, \ldots, M)$ in $\Phi\left(\mathbf{P}^{\mathbf{M}}\right)$. We see that the accuracy of $\hat{\theta}$ (i.e. $P_{\theta}^{-1}$ ) is the sum of the accuracy obtained during the initial identification experiment (i.e. $P_{\theta_{1}}^{-1}$ ) and of the increases of accuracy obtained after each local LTI experiment in the second LPV identification procedure.

As already mentioned, the second LPV identification procedure will be designed in an optimal way. More precisely, we will determine the number $M$ of experiments, the set $\mathbf{P}^{\mathbf{M}}$ of operating points and the set of spectra $\Phi\left(\mathbf{P}^{\mathbf{M}}\right)$ in such a way that the corresponding LPV identification experiment is the one corresponding to the least input energy $\mathcal{J}=N_{p} \sum_{m=1}^{M} \frac{1}{2 \pi} \int_{-\pi}^{\pi} \Phi_{u_{m}}(\omega) d \omega$ while guaranteeing that the uncertainty set $U$ defined with (46) is small enough to ensure $J_{w c}^{u b}<\eta_{\text {rob }}$ for the (LPI or LPV) controller $\hat{\bar{C}}(p(t))$ designed with $\bar{G}(T(p(t) \hat{\theta})$.

\subsection{Design of $\Phi\left(\mathrm{P}^{\mathrm{M}}\right)$ for given $M$ and $\mathrm{P}^{\mathrm{M}}$}

Like in [16], a first step towards the solution of the above-mentioned optimal experiment design problem is to consider the case where we have fixed a-priori the number $M$ of local LTI experiments in the second LPV identification experiment and the set $\mathbf{P}^{\mathbf{M}}$ of operating points where these local LTI experiments will be performed. The optimal experiment problem then consists in determining $\Phi\left(\mathbf{P}^{\mathbf{M}}\right)$ in such a way that $\mathcal{J}$ is minimized while guaranteeing that the uncertainty set $U$ defined with (46) is small enough to ensure $J_{w c}<\eta_{r o b}$. 
To solve this optimal experiment design problem, we first need to define a parametrization for the to-be-determined spectra $\Phi_{u_{m}}(m=1, \ldots, M)$. Here, we will use two types of parametrization. The first parametrization [21] corresponds to the spectrum of a signal that is generated by a white noise filtered by an arbitrary FIR filter of degree $L$ ( $L$ is an user-chosen parameter):

$$
\Phi_{u_{m}}(\omega)=c_{m, 0}+2 \sum_{i=1}^{L} c_{m, i} \cos (i \omega)
$$

The positivity of (47) for all $\omega$ can be imposed by a LMI constraint on the coefficients $c_{m, i}(i=0, \ldots, L)[21]$. With this parametrization, the cost function $\mathcal{J}$ of the optimal experiment design problem defined above is given by:

$$
\mathcal{J}=N_{p} \sum_{m=1}^{M} \frac{1}{2 \pi} \int_{-\pi}^{\pi} \Phi_{u_{m}}(\omega) d \omega=N_{p} \sum_{m=1}^{M} c_{m, 0}
$$

which is, as we can see, an affine function of the coefficients $c_{m, i}(i=0, \ldots, L)$ ( $m=1, \ldots, M)$ of the parametrization of the spectra. Using (46) and (11), we see that the inverse $P_{\theta}^{-1}$ of the covariance matrix of $\hat{\theta}$ is also an affine function of these coefficients.

The second spectrum parametrization considered in this paper corresponds to the spectrum of a multisine signal at fixed frequencies $\omega_{i}(i=0 \ldots L)$ but with unknown amplitudes [21]: $\Phi_{u_{m}}(\omega)=\pi \sum_{i=0}^{L} c_{m, i}\left(\delta\left(\omega-\omega_{i}\right)+\delta\left(\omega+\omega_{i}\right)\right)$. In this case, the positivity of $\Phi_{u_{m}}(\omega)$ for all $\omega$ can be imposed by the constraints $c_{m, i}>0(i=0, \ldots, L)$. The $\operatorname{cost} \mathcal{J}=N_{p} \sum_{m=1}^{M} \sum_{i=0}^{L} c_{m, i}$ and $P_{\theta}^{-1}$ are here also affine functions of the coefficients $c_{m, i}(i=0, \ldots, L)(m=1, \ldots, M)$.

\subsubsection{Non-convex optimal experiment design for the LPI and LPV cases}

When $M$ and $\mathbf{P}^{\mathbf{M}}$ are fixed, the optimal experiment design problem in both the LPI and LPV situations consists in determining the coefficients $c_{m, i}(i=0, \ldots, L)$ $(m=1, \ldots, M)$ of the spectra in $\Phi\left(\mathbf{P}^{\mathbf{M}}\right)$ using the following optimization problem:

$$
\begin{aligned}
& \min \mathcal{J} \\
& \text { over } A(\omega) \in \mathcal{A} \text { and } c_{m, i} \in \mathbf{R}(i=0, \ldots, L)(m=1, \ldots, M) \\
& \text { such that }\left(\begin{array}{c}
M\left(e^{j \omega}\right) \\
I
\end{array}\right){ }^{*} \mathcal{B}\left(A(\omega), \eta_{\text {rob }}^{2}\right)\left(\begin{array}{c}
M\left(e^{j \omega}\right) \\
I
\end{array}\right)<0 \quad \forall \omega
\end{aligned}
$$

The constraints of the above optimization problem have to be completed by the constraints on $c_{m, i}$ guaranteeing that $\Phi_{u_{m}}>0 \forall \omega(m=1, \ldots, M)$. In $(49), \mathcal{B}(.,$.$) is$ defined as in (35) where $\mathcal{A}$ is either $\mathcal{A}_{\bar{P}}$ in the LPV situation or $\mathcal{A}_{\mathbf{P}}$ in the LPI situation. Indeed, the existence of a frequency-dependent matrix $A(\omega) \in \mathcal{A}$ such that the constraint in (49) holds is equivalent to $J_{w c}^{u b}<\eta_{r o b}$ (see Propositions 1 and 4). Recall that the actual decision variables corresponding to $A(\omega)$ are given below Proposition 3 and note that, in $\mathcal{B}\left(A(\omega), \eta_{\text {rob }}^{2}\right)$, the term $A_{22}(\omega)$ contains $P_{\theta}^{-1}$ which is given in (46) and which is a function of the decision variables $c_{m, i}(i=0, \ldots, L)(m=1, \ldots, M)$.

There are a number of issues with this optimization problem. First, the constraint has to hold at an infinite number of frequencies. This issue can be solved using either 
the KYP lemma or, as we will do in this paper, a fine grid $\Omega$ of the interval $[0, \pi]$ (see also Section 4.4) and by replacing the constraint in (49) by the constraint (37) with $\gamma=\eta_{\text {rob }}^{2}$. Second, as in all optimal experiment design problem, (49) depends on a number of unknown variables. Indeed, $P_{\theta}^{-1}$ depends on the unknown true parameter vector $\theta^{0}$ (see (46) and (11)) and $M\left(e^{j \omega}\right)$ depends on the to-be-identified parameter vector $\hat{\theta}$ and the coefficients of the to-be-designed controller. This issue can, e.g., be resolved by replacing, in (49), these variables respectively by $\hat{\theta}_{1}$ and by the coefficients of the initial (LPI or LPV) controller $\hat{\bar{C}}_{1}(p(t))$. The final issue is related to the term $A_{22}(\omega)$ in $A(\omega)$ (see Propositions 2 and 3). In both the LPI and LPV situations, this term $A_{22}$ contains the Kronecker product of the decision variable $A_{0}(\omega)$ (also present in $A_{11}$ ) and of the expression of $P_{\theta}^{-1}$ as a function of the decision variables $c_{m, i}(i=0, \ldots, L)(m=1, \ldots, M)$. The optimization problem (49) is thus bilinear. To tackle this bilinearity, we will use the iterative approach inspired by the so called D-K iterations [36] and introduced in [5] (see also [9]). Before presenting this approach, we note that, if we arbitrarily choose the spectra $\Phi_{u_{m}}(m=1, \ldots, M)$, we can compute the corresponding $P_{\theta}$ via (46) and (11). With this $P_{\theta}$, we can compute $J_{w c}^{u b}$ via Proposition 4 and we can therefore verify whether $J_{w c}^{u b}<\eta_{r o b}$ or not. If that is the case, we will say that the spectra $\Phi_{u_{m}}(m=1, \ldots, M)$ are validated.

Algorithm 1. The algorithm is made up of an initialization step (step 0) and each iteration consists of two steps.

S.0. We initialize the algorithm by arbitrarily choosing the spectra $\Phi_{u_{m}}$ ( $m=$ $1, \ldots, M)$.

S.1. Using a subdivision algorithm, we determine, using the notion of validation defined above, the minimal positive scalar $\alpha \in \mathbf{R}$ such that the spectra $\alpha \Phi_{u_{m}}$ $(m=1, \ldots, M)$ remain validated. Denote this minimal $\alpha$ by $\alpha_{\text {min }}$. To validate $\alpha_{m i n} \Phi_{u_{m}}$, the optimization problem in Proposition 4 has been used. The corresponding decision variables are $\gamma$ and the free variables in $A(\omega)$. From those decisions variables, $A_{0}(\omega)$ is conserved for Step 2.

S.2. The optimal experiment design problem (49) is transformed into an LMI optimization problem by fixing the decision variable $A_{0}(\omega)$ to the one determined in Step 1. The solution of this transformed optimization problem define, via $c_{m, i}$, new spectra $\Phi_{u_{m}}$. These new spectra $\Phi_{u_{m}}$ can then be used in Step 1 for a new iteration.

The algorithm is stopped when the optimal cost $\mathcal{J}_{\text {opt }}$ in Step 2 no longer decreases significantly after each iteration. The optimal spectra $\Phi_{u_{m}}$ are then the ones corresponding to this last iteration (Step 1 is used a last time to further refine the solution).

Since the spectra $\Phi_{u_{m}}$ delivered by Algorithm 1 are validated, they are guaranteed to lead to a covariance matrix $P_{\theta}$ for which $J_{w c}^{u b}$ (computed with $\hat{\theta}_{1}$ and $\hat{\bar{C}}_{1}(p(t))$, the initial guesses, respectively, for $\theta^{0}, \hat{\theta}$ and for the to-be-designed controller) is smaller than $\eta_{\text {rob }}$. However, there is no guarantee that the corresponding cost $\mathcal{J}_{\text {opt }}$ is the smallest possible. Like in any non-convex optimization, it is advisable to initialize Algorithm 1 with spectra that are close to the ones solving the non-convex optimization problem described in (49). For the LPI case, this can be done by using a convex approximation of (49) which is described below. For the LPV case, this approach is not possible and a possible initialization is then the spectra used in the initial LPV experiment (the one yielding the initial guess $\hat{\theta}_{1}$ ). 


\subsubsection{Convex optimal experiment design based on a grid of $\mathcal{P}$}

In the LPI case, the approach presented in Section 4.5.2 allows one to approximate (49) by a convex alternative where the constraint on the upper bound on the worst case performance is replaced by a constraint on the lower bound of this worst case performance. For this purpose, we will thus have to approximate the scheduling space $\mathcal{P}$ by a fine grid $\mathcal{P}^{\text {grid }}$. This convex formulation consists in determining the coefficients $c_{m, i}$ $(i=0, \ldots, L)(m=1, \ldots, M)$ of the spectra in $\Phi\left(\mathbf{P}^{\mathbf{M}}\right)$ that minimize $\mathcal{J}$ under the constraint that we can find, for each $\omega$ in a fine grid $\Omega$ of $[0 \pi]$ and for each $\mathbf{p}$ in a fine grid $\mathcal{P}^{\text {grid }}$ of $\mathcal{P}$, a positive scalar $\tau(\omega, \mathbf{p})$ such that the following LMI holds:

$$
\tau(\omega, \mathbf{p})\left(\mathcal{E}(\omega, \mathbf{p})-\eta_{\text {rob }}^{2} \mathcal{E}_{\gamma}(\omega, \mathbf{p})\right)-\left(\begin{array}{cc}
P_{\theta}^{-1} & P_{\theta}^{-1} \hat{\theta} \\
P_{\theta}^{-1} \hat{\theta} & \chi-\hat{\theta}^{T} P_{\theta}^{-1} \hat{\theta}
\end{array}\right)<0
$$

Consequently, this LMI optimization problem contains one LMI for each pair $(\omega, \mathbf{p})$ such that $\omega \in \Omega$ and $\mathbf{p} \in \mathcal{P}^{\text {grid }}$. The decision variables are the coefficients $c_{m, i}$ $(i=0, \ldots, L)(m=1, \ldots, M)$ of the spectra in $\Phi\left(\mathbf{P}^{\mathbf{M}}\right)$ and the scalar coefficients $\tau(\omega, \mathbf{p})$ (one for each pair $(\omega, \mathbf{p})$ such that $\omega \in \Omega$ and $\mathbf{p} \in \mathcal{P}^{\text {grid }}$ ). In (50), the covariance matrix $P_{\theta}$ should be replaced by its expression as a function of the coefficients $c_{m, i}(i=0, \ldots, L)(m=1, \ldots, M)$. Here also the unknown quantities (such as $\hat{\theta}$ ) has to be replaced by initial guesses.

Imposing (50) for each $\omega \in \Omega$ and for each $\mathbf{p} \in \mathcal{P}^{\text {grid }}$ is equivalent to imposing that, for all $\omega \in \Omega$,

$$
J_{w c}(\omega, \mathbf{p})<\eta_{r o b} \quad \forall \mathbf{p} \in \mathcal{P}^{g r i d}
$$

with $J_{w c}(\omega, \mathbf{p})$ as defined in (43). Consequently, if the grid $\mathcal{P}^{\text {grid }}$ is sufficiently fine, the solution of this optimization problem will be very close to the solution of the original optimal experiment design problem. This solution can therefore be used as initialization for Algorithm 1. As will be shown in the numerical illustrations, Algorithm 1 may not be necessary since the solution obtained by the above procedure will be (approximately) equal to the optimal solution of (49).

\subsection{Selection of the optimal operating points}

The optimization problem (49) pertains to the determination of the optimal $\Phi\left(\mathbf{P}^{\mathbf{M}}\right)$ for given $M$ and $\mathbf{P}^{\mathbf{M}}$. Let us for further reference denote the optimal cost of (49) by $\mathcal{J}_{\text {opt }}\left(M, \mathbf{P}^{\mathbf{M}}\right)$. In this section, we will consider the more general optimal experiment design problem where $M$ and $\mathbf{P}^{\mathbf{M}}$ have to be determined together with $\Phi\left(\mathbf{P}^{\mathbf{M}}\right)$. Recall that $M, \mathbf{P}^{\mathbf{M}}$ and $\Phi\left(\mathbf{P}^{\mathbf{M}}\right)$ are the experimental conditions of a second LPV identification experiment (see Section 5.1). Like in [16], we will assume that the local LTI identification experiments of this second LPV identification experiment can only be performed at the $M_{\text {grid }}$ points in a fine grid $\mathbf{P}^{\text {grid }}=\left\{\mathbf{p}_{\mathbf{1}}, \ldots, \mathbf{p}_{\mathbf{M}_{\text {grid }}}\right\}$ of the continuous scheduling interval $\mathcal{P}=\left[-p_{\max }, p_{\max }\right]$. In other words, we assume that the set $\mathbf{P}^{\mathbf{M}}$ can only be chosen as a subset of $\mathbf{P}^{\text {grid }}$. Under this assumption, the optimal cost $\mathcal{J}_{\text {opt }}$ of the more general optimal experiment design problem will be the minimal value of $\mathcal{J}_{\text {opt }}\left(M, \mathbf{P}^{\mathbf{M}}\right)$ among all possible subsets $\mathbf{P}^{\mathbf{M}}$ of $\mathbf{P}^{\text {grid }}$.

In the sequel, we will show that $\mathcal{J}_{\text {opt }}$ is in fact given by $\mathcal{J}_{\text {opt }}\left(M_{\text {grid }}, \mathbf{P}^{\text {grid }}\right.$ ) (see 
also [16]). Let us for this purpose observe that the optimization problem (49) only depends, via $\mathcal{J}$ and $P_{\theta}^{-1}$, on the experimental conditions $M, \mathbf{P}^{\mathbf{M}}$ and $\Phi\left(\mathbf{P}^{\mathbf{M}}\right)$. Let us also observe that, for any (second) LPV identification experiment at the operating points in a strict subset $\mathbf{P}^{\mathbf{M}}$ of $\mathbf{P}^{\text {grid }}$, one can find a (mathematically) equivalent LPV experiment with local LTI experiments at all operating points in Pgrid that leads to the same cost $\mathcal{J}$ and the same $P_{\theta}^{-1}$. This mathematically equivalent experiment with $M_{\text {grid }}$ local LTI experiments is an experiment where $\Phi_{u_{m}}$ is chosen equal to zero for all $M_{\text {grid }}-M$ operating points that lie in $\mathbf{P}^{\text {grid }}$, but not in $\mathbf{P}^{\mathbf{M}}$. Both experiments have of course the same spectrum $\Phi_{u_{m}}$ for the common operating points. Conversely, a LPV identification experiment at all operating points in $\mathbf{P}^{\text {grid }}$, for which $\Phi_{u_{m}}=0$ at a certain number of operating points, is (mathematically) equivalent to an experiment at only those operating points for which $\Phi_{u_{m}} \neq 0$ (see [16] for the full discussion).

Based on the discussion above, it is clear that the minimal cost $\mathcal{J}_{\text {opt }}$ will thus be obtained by solving (49) (via Algorithm 1) for $M=M_{\text {grid }}$ and $\mathbf{P}^{\mathbf{M}}=\mathbf{P}^{\text {grid }}$. However, in general, the optimal second LPV identification experiment will not require $M_{\text {grid }}$ local LTI experiments since the optimization problem for $M=M_{\text {grid }}$ and $\mathbf{P}^{\mathbf{M}}=$ $\mathbf{P}^{\text {grid }}$ will generally lead to a sparse solution with a large number of spectra $\Phi_{u_{m}}$ equal to 0 . The optimal experiment will therefore be the (mathematically equivalent) LPV identification experiment with local LTI identification experiments at only those operating points in $\mathbf{P}^{\text {grid }}$ for which the corresponding spectra $\Phi_{u_{m}}$ are nonzero.

The tendency to obtain sparse solutions had already been observed in [16] where different (and much simpler) accuracy constraints were considered. It can be explained as follows. Since $\mathcal{J}$ must be minimized, power will only be injected at those operating points that allow to obtain the maximum information on $\theta^{0}$ and therefore, in turn, the smallest uncertainty set $U$. This phenomenon can presumably also be explained by the fact that the cost function $\mathcal{J}$ has a $l_{1}$-norm structure and it is frequently observed that such cost functions, when minimized under convex constraints ${ }^{10}$ generate a sparse solution (see, e.g., [32]).

Note that what has been said in this section for the optimal experiment design problem (49) also holds for the optimal experiment problem presented in Section 5.2.2.

\section{Numerical illustration 1}

\subsection{Description of the LPV system}

For this numerical illustration, we consider a true system $\bar{G}\left(T(p(t)) \theta^{0}\right)$ of the form (2) with $A\left(z, \zeta^{0}(p(t))=1+a_{1}^{0}(p(t)) z^{-1}\right.$ and $B\left(z, \zeta^{0}\right)=b_{0}^{0}(p(t)) z^{-1}$. The two parameters in these polynomials are supposed to have an affine dependence on the scheduling variable $p(t)\left(n_{p}=1\right): a_{1}^{0}(p(t))=-0.7+0.1 p(t)$ and $b_{0}^{0}(p(t))=8-1 p(t)$ with a scheduling interval $\mathcal{P}$ characterized by $p_{\max }=2$, i.e., $\mathcal{P}=[-2,2]$. The true parameter vector $\theta^{0}$ is therefore given by: $\theta^{0}=(-0.7,0.1,8,-1)^{T}$. We also suppose that the noise-free output $\breve{y}$ is perturbed as shown in (5)-(6) with a stochastic disturbance $v(t)$ characterized by $H(z)=\frac{1}{1-0.995 z^{-1}}$ and $\sigma_{e}^{2}=0.5$.

${ }^{10}$ Even though the constraint in (49) is not convex due to the Kronecker product of $P_{\theta}^{-1}$ and $A_{0}(\omega)$, the optimization problem is actually solved via Algorithm 1 where the constraint is convexified. 


\subsection{LPI situation}

\subsubsection{Initial identification and Robustness analysis}

Let us first consider the LPI situation. A first identification experiment is performed on the true system in order to obtain a model for the design of a LPI controller. This first identification experiment is made up of four local LTI experiments of duration $N_{p}=1000$ at the operating points $\mathbf{p}_{1}=-1.25, \mathbf{p}_{2}=-0.75, \mathbf{p}_{3}=-0.25$ and $\mathbf{p}_{4}=$ 0.75. The input spectra $\Phi_{u_{m}}$ for these four local LTI experiments are all given by: $\Phi_{u_{m}}(\omega)=0.05 \forall \omega(m=1, \ldots, 4)$. Following this procedure, we have obtained a first estimate $\hat{\theta}$ of $\theta^{0}$ together with its uncertainty ellipsoid $U$ defined as in (14) with the covariance matrix $P_{\theta}$ of $\hat{\theta}$ and with $\chi=9.5(\chi=9.5$ corresponds to $\beta=0.95)$.

With the model $G(z, T(\mathbf{p}) \hat{\theta})$, we design a LPI controller $\hat{C}(z, \mathbf{p})$ ensuring a certain nominal level of disturbance rejection and limited control efforts when applied to $G(z, T(\mathbf{p}) \hat{\theta})$, for $\mathbf{p} \in \mathcal{P}$. Focusing on the disturbance rejection and defining $W_{i}(z)=$ $\frac{0.1565-0.1416 z^{-1}}{1-0.995 z^{-1}}$, the nominal performance of the loop made up of the LPI controller $\hat{C}(z, \mathbf{p})$ and the model $G(z, T(\mathbf{p}) \hat{\theta})$ is given by $J_{n o m}=\sup _{\mathbf{p} \in \mathcal{P}}\left\|\frac{W_{i}(z)}{1+\hat{C}(z, \mathbf{p}) G(z, T(\mathbf{p}) \hat{\theta})}\right\|_{\infty}$. Using a procedure similar to the one in Section 4 , but with an uncertainty limited to $p$, an upper bound for the nominal performance can be evaluated. This upper bound for the nominal performance $J_{\text {nom }}$ is here equal to 0.2528 . The frequency-wise version of $J_{\text {nom }}$ given in the left hand side of (24) is represented in Figure 3 (blue dash-dotted curve).

Let us now analyze the robustness of the designed LPI controller over the uncertainty region $U$ and verify therefore whether (27) is satisfied when we choose $\eta_{r o b}=0.2654$ (i.e. we allow a $5 \%$-degradation with respect to the nominal performance: $0.2654=1.05 \times 0.2528$ ). For this purpose, we will follow the procedure of Section 4.5 and use Proposition 5 and Remark 1 to compute, at each frequency $\omega$ in a fine grid $\Omega$ of $[0, \pi]$, the upper bound $\tilde{J}_{w c}^{u b}(\omega)$ for the quantity $\tilde{J}_{w c}(\omega)$ defined in (41). The obtained upper bound $\tilde{J}_{w c}^{u b}(\omega)$ is represented in Figure 3 (red solid curve) and we observe that the robustness condition (27) is not respected since we do not have that $\tilde{J}_{w c}^{u b}(\omega)<\eta_{\text {rob }} /\left|W_{i}\left(e^{j \omega}\right)\right|$ at all frequencies.

As already mentioned, we verify the robustness of the controller based on an upper bound $\tilde{J}_{w c}^{u b}(\omega)$ of the actual worst case performance $\tilde{J}_{w c}(\omega)$. This could lead to conservatism if $\tilde{J}_{w c}^{u b}(\omega)$ is much larger than $\tilde{J}_{w c}(\omega)$. In order to verify that this conservatism remains limited, we have also computed, using the procedure of Section 4.5.2, the lower bound $\tilde{J}_{w c}^{l b}(\omega)$ for the quantity $\tilde{J}_{w c}(\omega)$. This lower bound is also represented in Figure 3 (green dashed curve) and we observe that the conservatism is indeed limited in this example since the lower and upper bounds are almost overlapping.

\subsubsection{Optimal experiment design}

As shown in the previous subsection, the initial uncertainty region $U$ is thus too large. We will therefore proceed to optimal experiment design to reduce the size of this uncertainty in an optimal way. We follow the procedure in Section 5 to design a second LPV identification experiment (with minimal input energy $\mathcal{J}$ ) which combined with the first one will yield a model with a smaller uncertainty region $U$ for the LPI controller designed based on this new model to be guaranteed to satisfy (27) with the new uncertainty region $U$. For this purpose, as proposed in Section 5.3, we suppose that the local LTI identification experiments of the second LPV identification experiment are all of duration $N_{p}=1000$ and that they can only be performed at the $M_{\text {grid }}=17$ 


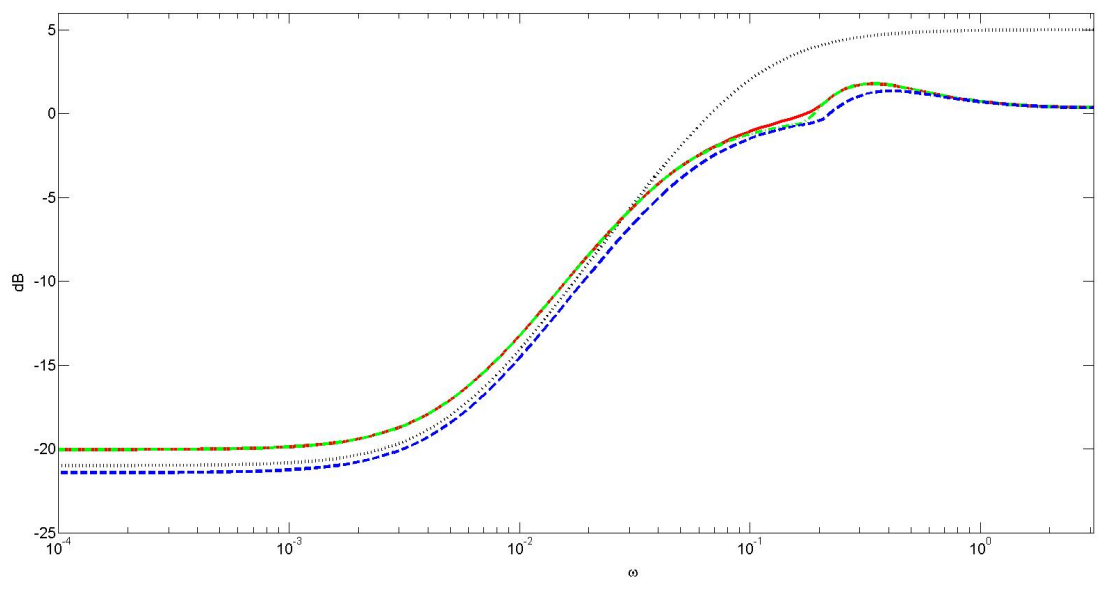

Figure 3.: LPI situation: desired performance $\eta_{r o b} /\left|W_{i}\left(e^{j \omega}\right)\right|$ (black dotted), $\tilde{J}_{w c}^{u b}(\omega)$ (red solid), $\tilde{J}_{w c}^{l b}(\omega)$ (green dashed-dotted) and the upper bound for the left hand side of (24) (blue dashed) as a function of the frequency $\omega$

operating points obtained by gridding the scheduling interval $\mathcal{P}$ with a fixed step of 0.25 , i.e., $\mathbf{P}^{\text {grid }}=\{-2,-1.75,-1.5, \ldots, 1.75,2\}$. We then solve the optimal experiment design problem (49) for $M=M_{\text {grid }}$ and $\mathbf{P}^{\mathbf{M}}=\mathbf{P}^{\text {grid }}$ and for $\eta_{\text {rob }}=0.2654$. The input spectra will be parametrized as in (47) with $L=12$ while the model and the controller obtained after the initial experiment (see Section 6.2.1) will be used in lieu of the unknown variables in (49). Due to the bilinearity of (49), Algorithm 1 is used to solve this optimization problem. To initialize this algorithm, we have considered both the initialization proposed in Section 5.2.2 as well as an initialization with the white noise spectra used in the initial LPV identification experiment (see Section 6.2.1). Both initializations here lead to the same (sub)optimum $\mathcal{J}_{\text {opt }}=\mathcal{J}_{\text {opt }}\left(M_{\text {grid }}, \mathbf{P}^{\text {grid }}\right)=103$ and to the same optimal spectra after one iteration of Algorithm 1. Further iterations do not decrease this cost. Algorithm 1 thus seems robust to its initialization in this example, but the advantage of the initialization of Section 5.2.2 is that the initialization spectra are (as expected) already approximately equal to the optimal spectra delivered by Algorithm 1 (i.e. the ones in Figure 4).

In addition, we observe that the obtained spectra $\Phi_{u_{m}}(m=1, \ldots, 17)$ are nonzero at only two values (i.e. $\mathbf{p}=-2$ and $\mathbf{p}=2$ ) of the 17 operating points in $\mathbf{P}^{\text {grid }}$. The nonzero spectra at $\mathbf{p}=-2$ and $\mathbf{p}=2$ are given in Figure 4. Using the same initial guesses as for solving (49) to evaluate the covariance matrix (46) of the uncertainty ellipsoid $U$ corresponding to the combination of the initial LPV experiment (see Section 6.1) and this optimal LPV experiment with $L=12$, we evaluate $\tilde{J}_{w c}^{u b}(\omega)$ for this new uncertainty $U$ (red solid curve in Figure 5 ) and we observe that, as expected, the robustness condition is now respected since $\tilde{J}_{w c}^{u b}(\omega)<\eta_{\text {rob }} /\left|W_{i}\left(e^{j \omega}\right)\right|$ at all frequencies.

In order to further validate our results, we perform the second LPV identification experiment on the true system by realizing the two local LTI identification experiments with input signals of duration $N_{p}=1000$ and of spectra as given in Figure 4. Combining this optimal experiment and the initial experiment, we can deduce a new estimate $\hat{\theta}$ and its covariance matrix (46) yielding the uncertainty ellipsoid $U$. Based on the new model $\bar{G}(T(\mathbf{p}) \hat{\theta})$ and with the same LPI control design method as in Section 6.1 , we can deduce a new LPI controller $\hat{C}(z, \mathbf{p})$. The robustness of this new LPI 


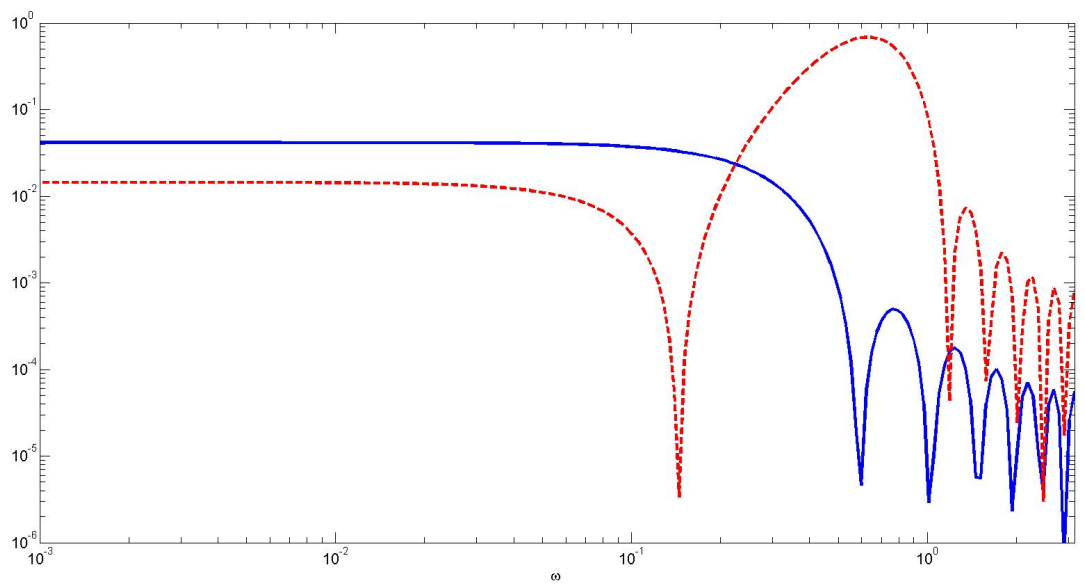

Figure 4.: LPI situation: optimal power spectra $\Phi_{u}$ for $\mathbf{p}=-2$ (blue solid) and for $\mathbf{p}=2$ (red dashed)

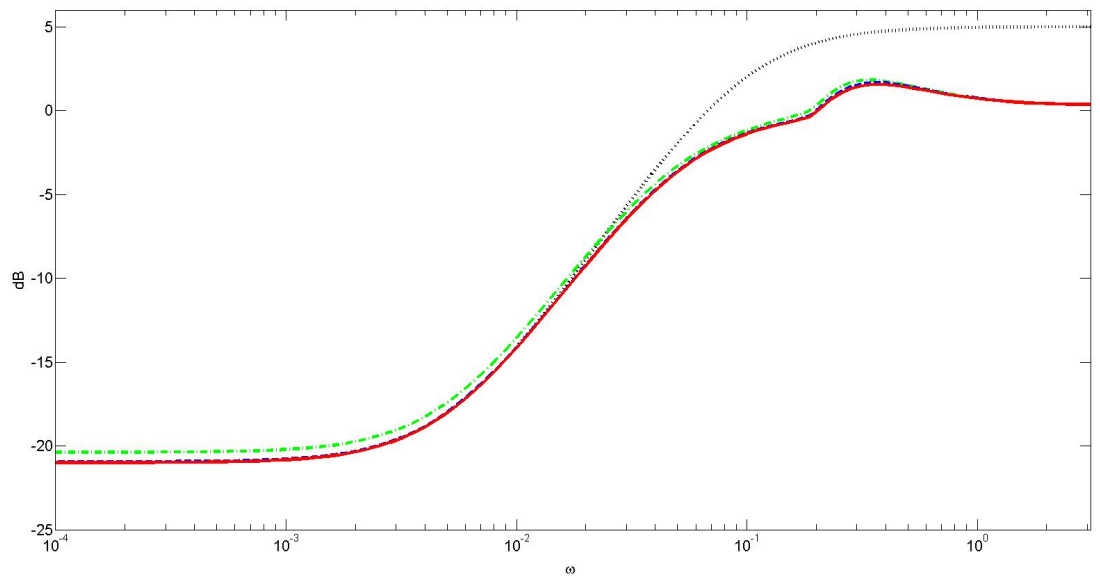

Figure 5.: LPI situation: desired performance $\eta_{r o b} /\left|W_{i}\left(e^{j \omega}\right)\right|$ (black dotted), expected worst case performance $\tilde{J}_{w c}^{u b}(\omega)$ after optimal experiment design and using the initial guesses (red solid), obtained worst case performance $\tilde{J}_{w c}^{u b}(\omega)$ after identification and redesign of the controller (blue dashed), worst case performance $\tilde{J}_{w c}^{u b}(\omega)$ corresponding to a (non-optimized) second LPV experiment using the global approach (green dashdotted) 
controller over the new uncertainty region $U$ is analyzed by computing $\tilde{J}_{w c}^{u b}(\omega)$ at each frequency $\omega$ (blue dashed curve in Figure 5) and we observe that, even if initial guesses were used for the design of the optimal spectra, the robustness condition is, as desired, respected since $\tilde{J}_{w c}^{u b}(\omega)<\eta_{r o b} /\left|W_{i}\left(e^{j \omega}\right)\right|$ at all frequencies.

The cost $\mathcal{J}$ of the initial LPV identification experiment (see Section 6.1) is equal to 200 while we have seen that the cost of the second LPV identification experiment (the one that has been optimized) is equal to 103. Using the proposed procedure, the total identification cost to obtain a sufficiently accurate model for LPI control is thus $\mathcal{J}=200+103=303$. Now, using the experimental conditions of the initial experiment (i.e. four LTI identification experiments at $\mathbf{p}_{1}=-1.25, \mathbf{p}_{2}=-0.75, \mathbf{p}_{3}=-0.25$ and $\mathbf{p}_{4}=0.75$ with white noise inputs having the same power for each of them), we would have needed a cost of 2075 to obtain the same result. This clearly show the benefit of optimizing the second LPV identification experiment.

\subsubsection{Local vs. global LPV identification}

The second LPV identification experiment that has been optimized in the previous subsection is based on the local approach for LPV identification. We will now compare this optimal local experiment with a global experiment having the same cost $\mathcal{J}=103$. In the global experiment, we will collect 2000 samples of input and output data (the optimal local LPV experiment consists of two times a local experiment of duration $N_{p}=1000$ ). The output data are obtained by applying, for $t=1, \ldots, 2000$, a white noise input $u(t)$ of variance 103/2000 (the cost of this global experiment is thus also equal to $\mathcal{J}=103$ ) while modifying $p(t)$ as a ramp going from $p(t)=-2$ at $t=1$ to $p(t)=2$ at $t=2000$. The covariance matrix corresponding to this global experiment will be estimated using the inverse of the Fisher information matrix and is combined with the information gathered during the initial experiment (like for the optimal local LPV experiment). We then compute $\tilde{J}_{w c}^{u b}(\omega)$ corresponding to this case (see the green dashed-dotted line in Figure 5) and we observe that the desired robust performance is not attained in this case. If we compare this green curve in Figure 5 with the red solid curve in Figure 3, we can also conclude that the amount of extra information brought by this non-optimized global LPV experiment is really limited.

\subsection{LPV situation}

\subsubsection{Initial identification and Robustness analysis}

Let us now consider the LPV situation. Since LPV control is more stringent, we decide to increase the power of the first LPV identification experiment with respect to the LPI case. In other words, the initial identification experiment is also made up of four local LTI experiments of duration $N_{p}=1000$ at the operating points $\mathbf{p}_{1}=-1.25, \mathbf{p}_{2}=-0.75, \mathbf{p}_{3}=-0.25$ and $\mathbf{p}_{4}=0.75$, but the input spectra $\Phi_{u_{m}}$ for these four local LTI experiments are now all given by: $\Phi_{u_{m}}(\omega)=0.15 \forall \omega(m=$ $1, \ldots, 4)$. With the model $\bar{G}(T(p(t)) \hat{\theta})$ of the LPV system, we design an LPV controller $\hat{\bar{C}}(p(t))$ ensuring a certain nominal level of disturbance rejection and limited control efforts when applied to $\bar{G}(T(p(t)) \hat{\theta})$ for $p \in \bar{P}$. Focusing here also on the disturbance rejection, the nominal performance of the LPV controller $\hat{\bar{C}}(p(t))$ can be defined as $J_{\text {nom }}=\sup _{p \in \bar{P}}\left\|\hat{\bar{S}}(p(t)) W_{i}\right\|_{\mathcal{L}_{2}}$ with $\hat{\bar{S}}(p(t))$ as defined in Section 3.2 and with the same $W_{i}(z)$ as in the LPI case. We can here also evaluate an upper bound for $J_{\text {nom }}$ 
which is here equal to 0.8557 . As expected since $\mathbf{P} \subset \bar{P}$, we observe that the level of (nominal) performance is lower in the LPV case.

We now analyze the robustness of the designed LPV controller over the uncertainty region $U$. For this purpose, we will verify whether $(27)$ is satisfied when we choose $\eta_{r o b}=0.8985$ (i.e. we allow a $5 \%$-degradation with respect to the nominal performance: $0.8985=1.05 \times 0.8557)$. We follow the procedure of Section 4.4 and use Proposition 4 to compute the upper bound $J_{w c}^{u b}$ for the quantity $J_{w c}$ defined in (26). The obtained upper bound $J_{w c}^{u b}$ is here equal to 1.0597 which is obviously larger than $\eta_{\text {rob }}=0.8985$.

\subsubsection{Optimal experiment design}

Like in Section 6.2.2, we will here also follow the procedure in Section 5 to design a second LPV identification experiment (with minimal input energy $\mathcal{J}$ ) which combined with the first one will yield a model with a smaller uncertainty region $U$ for the LPV controller designed based on this new model to be guaranteed to satisfy (27) with the new uncertainty region $U$. Using the same settings ${ }^{11}$ as for the LPI situation, we perform ten iterations of Algorithm 1 leading to an optimal second LPV identification experiment with a cost $\mathcal{J}_{\text {opt }}=165$ with, like in the LPI case, two local LTI identification experiments at the operating points $\mathbf{p}=-2$ and $\mathbf{p}=2$ and with similar spectra. It is to be noted that Algorithm 1 allowed us to reduce the cost from 197 (after the first iteration) to 165 (after the tenth iteration).

Using the procedure proposed in this paper, the total cost required to obtain a sufficiently accurate model for LPV control is thus $\mathcal{J}=600+165=765$. The benefit of optimizing the second LPV identification experiment can also in this case be easily evidenced: using the experimental conditions of the initial experiment (i.e. four LTI identification experiments at $\mathbf{p}_{1}=-1.25, \mathbf{p}_{2}=-0.75, \mathbf{p}_{3}=-0.25$ and $\mathbf{p}_{4}=0.75$ with white noise inputs having the same power for each of them), we would have needed a cost of 3393 to obtain the same result.

We then perform a second LPV identification experiment on the true system by realizing the two local LTI identification experiments with input signals of duration $N_{p}=1000$ and having the optimal spectra determined by the experiment design. Combining this optimal experiment and the initial experiment, we can deduce a new estimate $\hat{\theta}$ and its covariance matrix (46) yielding the uncertainty ellipsoid $U$. Based on the new model $\bar{G}(T(\mathbf{p}) \hat{\theta})$ and with the same LPV control design method as in Section 6.3.1, we can deduce a new LPV controller $\hat{\bar{C}}(p(t))$. The robustness of this new LPV controller over the new uncertainty region $U$ is analyzed by computing $J_{w c}^{u b}$. We obtain $J_{w c}^{u b}=0.8972 \approx \eta_{r o b}$ showing that the new uncertainty is small enough to guarantee the robustness condition.

\section{Numerical illustration 2}

Accelerometers [14] are sensors that are generally operated in closed loop and whose dynamics depend on the temperature. An accelerometer can be seen as a mass whose position $y$ is influenced by the total force on this mass. This total force is the sum of the force $v$ induced by the acceleration and a counter-force $u$ generated by the control system. The control system is designed in such a way that the total force is

${ }^{11}$ with an initialization of Algorithm 1 using the same spectra as in the initial LPV experiment. 
equal to zero (i.e. the mass position remains close to zero). The applied counter-force is therefore a measure of the acceleration.

The dynamical relation between the total force (i.e. $u+v)$ and the mass position can be described by a second-order differential equation which is dependent on the temperature (i.e. the temperature is the scheduling variable $p$ of this example). At a given frozen temperature, the transfer function between the total force and the mass position is generally a second-order transfer function with a resonance whose frequency depends on the temperature. It will be supposed that the temperature will vary slowly and that the accelerometer operation will therefore be close to the LPI situation. The LPI controller will be designed in such a way that, for each temperature in the working range, the output of the controller $u$ (i.e. the counter-force) is close to $-v$ for acceleration force $v$ having a frequency content up to a certain frequency. The force induced by the acceleration is thus considered as an (input) disturbance in the control problem and the control objective can be achieved by imposing a weighting on the sensitivity function (which is the transfer function between $v$ and the difference between $u$ and $-v$ ). $\operatorname{tion}^{12}$ :

We will illustrate the accelerometer using the following continuous-time descrip-

$$
\ddot{y}(t)+\zeta_{1}^{0}(p(t)) \dot{y}(t)+\zeta_{2}^{0}(p(t)) y(t)=\zeta_{3}^{0}(p(t))(u(t)+v(t))
$$

where $\zeta_{1}^{0}(p(t))=\theta_{1}^{0}+\theta_{2}^{0} p(t), \zeta_{2}^{0}(p(t))=\theta_{3}^{0}+\theta_{4}^{0} p(t)+\theta_{5}^{0} p^{2}(t)$ and $\zeta_{3}^{0}(p(t))=\theta_{6}^{0}$ with $\theta^{0}=\left(\theta_{1}^{0}, \ldots, \theta_{6}^{0}\right)^{T}=(0.3,0.1,0.5625,0.375,0.0625,1)^{T}$. The scheduling variable $p$ represents here the normalized temperature varying in the working range $\mathcal{P}=\left[\begin{array}{ll}-1 & 1\end{array}\right]$. If we compare the transfer functions between $u+v$ and $y$ for different operating points $\mathbf{p}$ in the working range, the resonance frequency $\sqrt{\zeta_{2}^{0}(\mathbf{p})}$ varies between 0.5 $\mathrm{rad} / \mathrm{s}($ when $\mathbf{p}=-1)$ and $1 \mathrm{rad} / \mathrm{s}($ when $\mathbf{p}=1)$.

We want to design an LPI controller for this accelerometer. The main control objective will be to guarantee a sensitivity function with a minimal gain of 0.02 in the frequency range [ $\left[\begin{array}{ll}0 & 0.075\end{array}\right]$ which we suppose to be the main bandwidth of the to-be-measured acceleration $v$. This performance objective can, e.g., be expressed via the sensitivity weighting $W_{i}=\frac{100 s^{2}+20 s+1}{0.25 s^{2}+s+1}$ and via $\eta_{r o b}=0.011$ as can be seen in Figure 6.

To design the LPI controller and guarantee this control objective even in the presence of modeling error, we need a sufficiently accurate estimate of the true parameter vector $\theta^{0}$. This estimate will be deduced using the procedure of Section 2 under no acceleration (i.e. $v=0$ ) and using multisine counter-force $u$ at different operating points $\mathbf{p}$. We suppose that the output data $y$ are sampled with a sampling time of $0.31 s$ and perturbed by a measurement noise which is a white noise of variance $\sigma_{e}^{2}=0.5$. We also suppose that the duration of the local LTI experiments is $N_{p}=1000$.

Since the dynamics (52) is only valid for relatively small total force, the optimal experiment design procedure aiming at minimizing the excitation energy

\footnotetext{
${ }^{12}$ The results of this paper can be easily extended to the continuous-time situation using the same philosophy as in, e.g., [27]
} 
$\mathcal{J}$ (see Section 5) is certainly relevant here. Since we are considering the LPI case, we will first use the procedure of Section 5.2.2 to determine the optimal operating points of which the local identification experiments have to be performed and the (multisine) excitation signals to be applied at those operating points. As mentioned in Section 5.2.1. the experiment design procedure requires an initial estimate $\hat{\theta}_{1}$. We will here suppose that this initial estimate ${ }^{13}$ is given as: $\hat{\theta}_{1}=(0.2493,0.0202,0.5605,0.2996,-0.0374,0.9636)^{T}$. Using this initial estimate, we can design an initial LPI controller which ensures a nominal performance $\eta_{\text {nom }}=0.01<\eta_{\text {rob }}$ with the weighting $W_{i}$ defined above (see blue-dashed curve in Figure 6).

Moreover, we choose $\mathbf{P}^{\text {grid }}$ as $(-1,-0.9,-0.8, \ldots, 0.9,1)\left(M_{\text {grid }}=21\right)$. The spectra $\Phi_{u_{m}}\left(m=1, \ldots, M_{\text {grid }}\right)$ are parametrized to represent multisines containing ten equally spaced frequencies $\omega_{i}$ ranging from 0.1 to $1 \mathrm{rad} / \mathrm{s}$. By solving the optimal experiment design problem ${ }^{14}$ of Section 5.2.2 for $M=M_{\text {grid }}$ and $\mathbf{P}^{\mathbf{M}}=\mathbf{P}^{\text {grid }}$, we obtain a sparse solution with $M=3$ local LTI experiments ${ }^{15}$ at $\mathbf{p}=-1, \mathbf{p}=-0.9$ and $\mathbf{p}=1$. The obtained multisines are also sparse, i.e., for each operating point, only two amplitudes out of the ten are significant. It is to be noted that, for each of the three operating points, one of these two frequencies is at the resonance frequency of the corresponding local LTI system (which is logical since we try to minimize the input energy). The optimal cost $\mathcal{J}_{\text {opt }}$ is equal to 156.4.

If we evaluate $P_{\theta}$ using the same initial guesses as in the optimal experiment design procedure, the lower bound $\tilde{J}_{w c}^{l b}(\omega)$ for $\tilde{J}_{w c}(\omega)$ is guaranteed to satisfy $\tilde{J}_{w c}^{l b}(\omega)<$ $\eta_{\text {rob }} /\left|W_{i}(j \omega)\right|$ at all frequencies. Instead of using Algorithm 1 to refine this optimal experiment, we here instead just verify that the grid $\mathcal{P}^{\text {grid }}$ has been chosen sufficiently fine to also guarantee that $\tilde{J}_{w c}^{u b}(\omega)<\eta_{r o b} /\left|W_{i}(j \omega)\right|$ at all frequencies $\left(\tilde{J}_{w c}^{u b}(\omega)\right.$ computed using the procedure of Section 4.5.1 and the same $\left.P_{\theta}\right)$. We observe that it is here the case (see the red-solid curve in Figure 6) and we thus conclude that further refinement via Algorithm 1 is not necessary in this example.

In order to further validate our results, we really perform the optimal LPV identification experiment on the true system by realizing the three local LTI identification experiments with input signals of duration $N_{p}=1000$ and corresponding to the optimal multisines. This delivers an estimate $\hat{\theta}$ and its covariance matrix (46) yielding the uncertainty ellipsoid $U$. Based on this estimate and with the same LPI control design method, we can deduce a new LPI controller $\hat{C}(z, \mathbf{p})$. The robustness of this new LPI controller over the new uncertainty region $U$ is analyzed by computing $\tilde{J}_{w c}^{u b}(\omega)$ at each frequency $\omega$ (blue dashed curve in Figure 7) and we observe that, even if initial guesses were used for the design of the optimal spectra, the robustness condition is, as desired, respected (modulo some numerical approximations/errors in low frequencies).

\section{Concluding remarks}

This paper constitutes a first step in connecting LPV control design and LPV system identification. More precizely, this paper extends to the LPV case the results of robustness analysis and of optimal experiment design for robust control that had been developed in $[7,10,5]$ in the LTI case. In this paper, we have nevertheless restricted

\footnotetext{
${ }^{13}$ For the sake of brievity, we will here suppose that $P_{\theta_{1}}^{-1}=0$.

${ }^{14} \mathrm{We}$ also add an accuracy constraint for the accuracy of each local estimate.

${ }^{15} M=3$ is the minimal number of local LTI experiments to obtain a consistent estimate $\hat{\theta}$.
} 


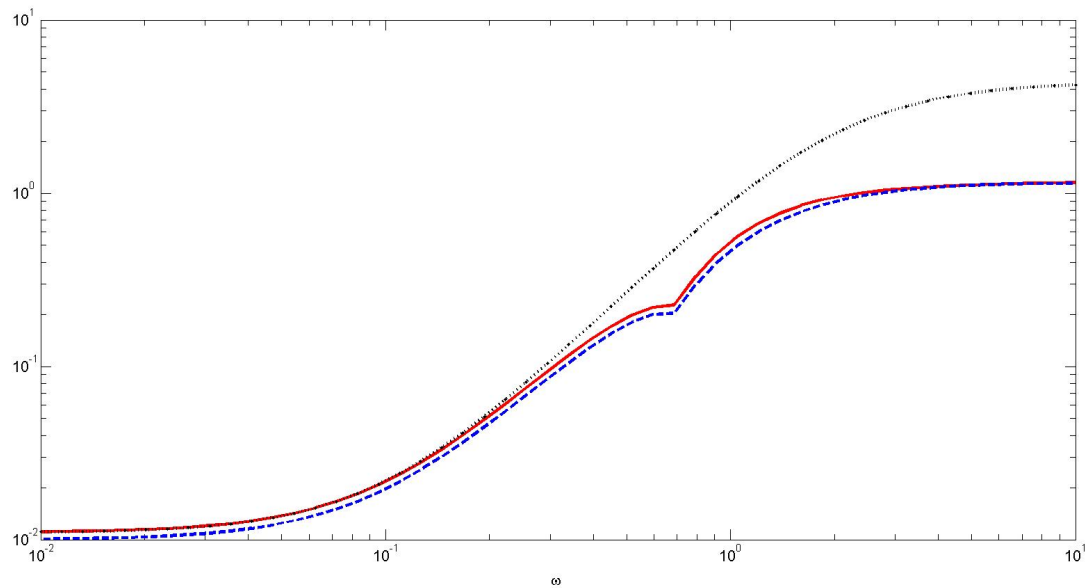

Figure 6.: Desired performance $\eta_{r o b} /\left|W_{i}(j \omega)\right|$ (black dotted), expected worst case performance $\tilde{J}_{w c}^{u b}(\omega)$ after optimal experiment design and using the initial guesses (red solid) and the upper bound for the left hand side of (24) (blue dash-dotted) as a function of the frequency $\omega$

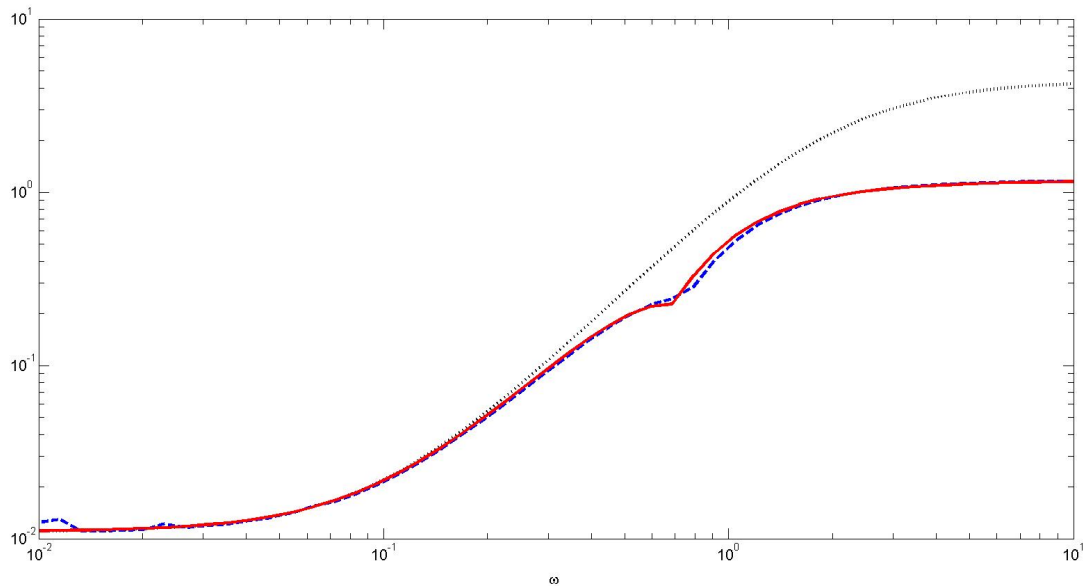

Figure 7.: Desired performance $\eta_{r o b} /\left|W_{i}(j \omega)\right|$ (black dotted), expected worst case performance $\tilde{J}_{w c}^{u b}(\omega)$ after optimal experiment design and using the initial guesses (red solid), obtained worst case performance $\tilde{J}_{w c}^{u b}(\omega)$ after identification and redesign of the controller (blue dashed) 
attention to LPV systems where the scheduling variable is considered as an external variable. Future work will consider the interesting case of quasi- $L P V$ systems where the scheduling variable can also be an internal variable of the system (e.g. the input or one of the elements of the state vector). We will also consider an interesting intermediary situation between the LPI and the LPV situations as they were defined in this paper, i.e., the case where the scheduling variable $p$ can vary over time, but with a limited rate of variation. Finally, we will also consider the case of optimal LPV experiment design in a closed-loop setting, i.e., the identification experiment has to be performed when the LPV system is already operated with an LPV controller.

\section{References}

[1] P. Apkarian and P. Gahinet. A convex characterization of gain-scheduled $H_{\infty}$ controllers. IEEE Transactions on Automatic Control 40(5), pp. 853-864, 1995.

[2] P. Apkarian, P. Gahinet, and J.M. Biannic. Gain-scheduled $H_{\infty}$ control of missile via linear matrix inequalities. Journal of Guidance, Control and Dynamics, vol 28 no 3, 1994.

[3] A.A. Bachnas, R. Tòth, J.H.A. Ludlage, and A. Mesbah. A review on data-driven linear parameter-varying modeling approaches: A high-purity distillation column case study. Journal of Process Control Vol 24, 272-285, 2014.

[4] B. Bamieh and L. Giarré. Identification of linear parameter varying models. Int. Journal of Robust and Nonlinear Control, Vol. 12, pp 841-853, 2002.

[5] M. Barenthin, X. Bombois, H. Hjalmarsson, and G. Scorletti. Identification for control of multivariable systems: controller validation and experiment design via LMIs. Automatica, 44(12):3070-3078, 2008.

[6] P.A. Bliman. Stabilization of LPV systems. In In Proc. IEEE Conf. on Decision and Control, pp. 6103-6108, 2003.

[7] X. Bombois, M. Gevers, G. Scorletti, and B.D.O. Anderson. Robustness analysis tools for an uncertainty set obtained by prediction error identification. Automatica, 37(10):1629-1636, 2001.

[8] X. Bombois, H. Hjalmarsson, and G. Scorletti. Identification for robust $H_{2}$ deconvolution filtering. Automatica, 46(3):577-584, 2010.

[9] X. Bombois, A. Korniienko, H. Hjalmarsson, and G. Scorletti. Optimal identification experiment design for the interconnection of locally controlled systems. Automatica, 89:169-179, 2018.

[10] X. Bombois, G. Scorletti, M. Gevers, P.M.J. Van den Hof, and R. Hildebrand. Least costly identification experiment for control. Automatica, 42(10):1651-1662, 2006.

[11] S. Boyd, L. El Ghaoui, E. Feron, and V. Balakrishnan. Linear Matrix Inequalities in Systems and Control Theory, volume 15 of Studies in Appl. Math. SIAM, Philadelphia, June 1994.

[12] A. Dankers, R. Tòth, P. Heuberger, X. Bombois, and P. Van den Hof. Informative data and identfiability for lpv-arx prediction error identification. In In Proc. IEEE Conference on Decision and Control, pages 799-804, 2011.

[13] M. Dinh, G. Scorletti, V. Fromion, and E. Magarotto. Parameter dependent $H_{\infty}$ control by finite dimensional lmi optimization. Int. J. Robust Nonlinear Control, 15:383-406, 2005.

[14] M. Elwenspoek and R. Wiegerink. Mechanical microsensors. Springer Science \& Business Media, 2012. 
[15] M. Gevers. Identification for control: from the early achievements to the revival of experiment design. European Journal of Control, 11:1-18, 2005.

[16] D. Ghosh, X. Bombois, J. Huillery, G. Scorletti, and G. Mercere. Optimal identification experiment design for LPV systems using the local approach. Automatica, 87:258-266, 2018.

[17] Marion Gilson, Vincent Laurain, Hugues Garnier, Sylvain Payraudeau, and Caroline Grégoire. A new data-based modelling method for identifying parsimonious nonlinear rainfall/flow models. Journal Européen des Systèmes Automatisés, Vol. 6(7), pp.878-888, 2011.

[18] K.C. Goh and M.G. Safonov. Robust analysis, sectors and quadratic functionals. In IEEE, editor, Proc. IEEE Conf. on Decision and Control, New Orleans, Louisiana, 1995.

[19] H. Hjalmarsson. System identification of complex and structured systems. European Journal of Control, 15(3-4):275-310, 2009.

[20] T. Iwasaki and G. Shibata. LPV system analysis via quadratic separator for uncertain implicit systems. IEEE Transactions on Atomatic Control, Vol. 46, pp. 1195-1208, 2001.

[21] H. Jansson and H. Hjalmarsson. Input design via LMIs admitting frequencywise model specifications in confidence regions. IEEE Transactions on Automatic Control, Vol 50 (10), pp. 1534-1549, 2005.

[22] Vincent Laurain, Marion Gilson, Roland Toth, and Hugues Garnier. Refined instrumental variable methods for identification of LPV Box-Jenkins models. Automatica, Vol. 46(6), pp. 959-967, 2010.

[23] Q. Liu, J. Gross, S. Pfeiffer, and H. Werner. A local approach for the LPV identification of an actuated beam using piezoelectric actuators and sensors. Mechatronics, Vol. 24, pp. 289-297, 2014.

[24] L. Ljung. System Identification - Theory for the User, 2nd Edition. Prentice-Hall, Upper Saddle River, NJ, 1999.

[25] A. Megretski and A. Rantzer. System analysis via integral quadratic constraints. IEEE Transactions on Automatic Control, 42:819-830, 1997.

[26] Harald Pfifer and Peter Seiler. Robustness analysis of linear parameter varying systems using integral quadratic constraints. In American Control Conference, Portland, USA, pp. 4476-4481, 2014.

[27] M. Potters, M. Mansoori, X. Bombois, J. Jansen, and P. Van den Hof. Optimal input experiment design and parameter estimation in core-scale pressure oscillation experiments. Journal of Hydrology, 534:534-552, 2016.

[28] M. G. Safonov. Stability and Robustness of Multivariable Feedback Systems. MIT Press, Cambridge, 1980.

[29] G. Scorletti and L. El Ghaoui. Improved LMI conditions for gain scheduling and related problems. International Journal of Robust and Nonlinear Control, 8(10):845-877, August 1998.

[30] M. Steinbuch, M.J.G. van de Molengraft, P.S.C. Heuberger, and A.J. Van der Voort. Experimental modelling and LPV control of a motion system. In Proceedings of the American Control Conference, pp. 1374- 1379, 2003.

[31] R Tòth. Modeling and identification of Linear Parameter Varying Systems. Springer, Berlin, 2010.

[32] Joel A. Tropp. Just relax: Convex programming methods for identifying sparse signals in noise. IEEE Transactions on Information Theory, Vol 52, pp. 1030$1051,2006$.

[33] M. G. Wassink, M. van de Wal, C. Scherer, and O. Bosgra. LPV control for a 
wafer stage: beyond the theoretical solution. Control Engineering Practice, Vol 13, pp. 231-245, 2005.

[34] J. C. Willems. The Analysis of Feedback Systems, volume 62 of Research Monographs. MIT Press, Cambridge, Massachusetts, 1969.

[35] W. Xie. $\mathrm{H}_{2}$ gain scheduled state feedback for LPV system with new LMI formulation. IEEE Proc. Control Theory Appl., Vol 152(6), pp.693-697, 2005.

[36] K. Zhou and J. Doyle. Essentials of Robust Control. Prentice Hall, Upper Saddle River, New Jersey, 1998.

\section{Appendix A. Description of the LPV system}

\section{A.1. Example of LFT representations}

For this example, we consider a true system $\bar{G}(T(p(t)) \theta)$ given by $\left(n_{p}=n_{a}=n_{b}=1\right)$ :

$$
\breve{y}(t)=-\left(\theta_{1}+\theta_{2} p(t)\right) \breve{y}(t-1)+\left(\theta_{3}+\theta_{4} p(t)\right) u(t-1)
$$

with $\theta=\left(\begin{array}{llll}\theta_{1} & \theta_{2} & \theta_{3} & \theta_{4}\end{array}\right)^{T}$. This relation can be represented as in Figure A.1. In this figure, we see that:

$$
\underbrace{\left(\begin{array}{l}
s_{1} \\
s_{2} \\
s_{3}
\end{array}\right)}_{=s_{G}}=\operatorname{bdiag}(p(t), \theta, \theta) \underbrace{\left(\begin{array}{l}
q_{1} \\
q_{2} \\
q_{3}
\end{array}\right)}_{=q_{G}}
$$

and we have also that:

$$
\left(\begin{array}{c}
q_{1} \\
q_{2} \\
q_{3} \\
\breve{y}
\end{array}\right)=\left(\begin{array}{cccc}
0 & -e_{2} & e_{4} & 0 \\
z^{-1} & -e_{1} z^{-1} & e_{3} z^{-1} & 0 \\
0 & 0 & 0 & z^{-1} \\
1 & -e_{1} & e_{3} & 0
\end{array}\right)\left(\begin{array}{c}
s_{1} \\
s_{2} \\
s_{3} \\
u
\end{array}\right)
$$

where $e_{i}(i=1, \ldots, 4)$ is a row vector of dimension 4 where the $i^{\text {th }}$ entry is equal to 1 and where all other entries are equal to zero. We have thus found that $\bar{G}(T(p(t)) \theta)$ is an LFT in $\operatorname{bdiag}(p(t), \theta, \theta)=\operatorname{bdiag}\left(p(t) I_{1}, I_{2} \otimes \theta\right)\left(n_{p_{G}}=1\right.$ and $\left.n_{\theta_{G}}=2\right)$. An LFT in $\operatorname{bdiag}(p, \delta \theta, \delta \theta)$ can then be also easily deduced by noticing that $\operatorname{bdiag}(p(t), \theta, \theta)$ can be written as an LFT in $\operatorname{bdiag}(p(t), \delta \theta, \delta \theta)$.

For this LPV system, the transfer function corresponding to a given operating point $\mathbf{p}$ is:

$$
\bar{G}(T(\mathbf{p}) \theta)=\frac{\left(\theta_{3}+\theta_{4} \mathbf{p}\right) z^{-1}}{1+\left(\theta_{1}+\theta_{2} \mathbf{p}\right) z^{-1}}=\frac{Z_{N}(z, \mathbf{p}) \theta}{1+Z_{D}(z, \mathbf{p}) \theta}
$$

with $Z_{N}(z, \mathbf{p})=\left(0,0, z^{-1}, \mathbf{p} z^{-1}\right)$ et $Z_{N}(z, \mathbf{p})=\left(z^{-1}, \mathbf{p} z^{-1}, 0,0\right)$. 


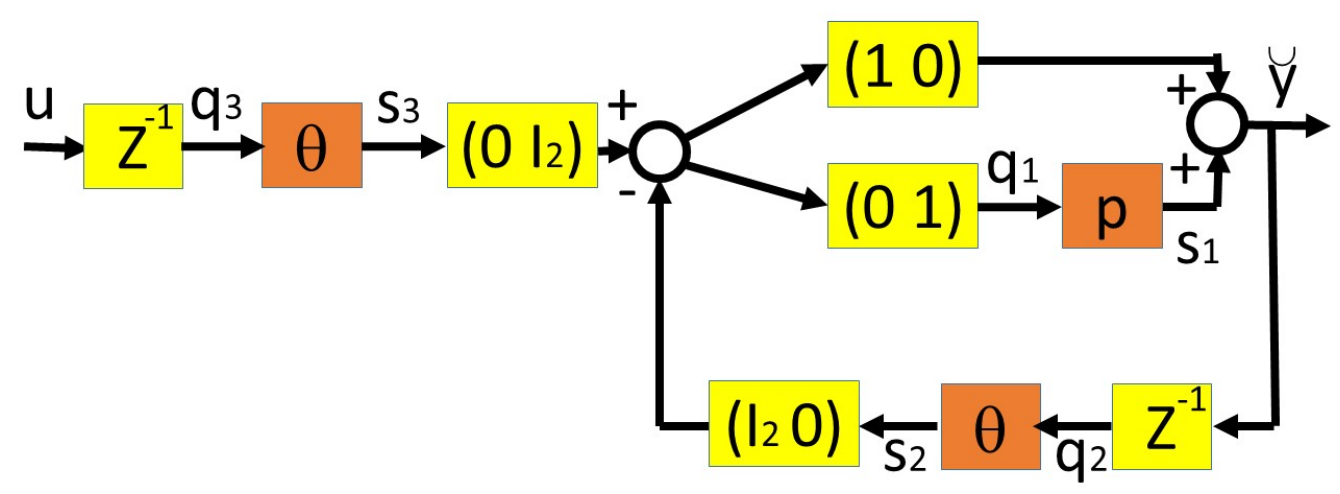

Figure A1.: Representation of the LPV system considered in Appendix A.1

\section{A.2. State-space representation vs. IO representation of the LPV system}

We have chosen to represent the LPV system using an input-output (IO) representation (see (2)). The LPI and LPV control design methods $[13,29]$ require a LPV system in state-space representation in which the coefficients of the state-space matrices are rational in the scheduling variable $p$. Canonical forms can be used to transform the IO representation (2) into a state-space representation. In order to show that, let us consider the type of IO representation introduced in Section 2.1:

$$
A(z, \zeta(p(t))) \breve{y}(t)=B(z, \zeta(p(t))) u(t)
$$

This representation holds both for the true system and for the model. For simplicity, we will suppose that the orders of the polynomials $A(z, \zeta)$ and $B(z, \zeta)$ are equal to $n_{a}=n_{b}+1 \triangleq \tilde{n}$ and that the delay $n_{k}=1$, i.e., $A(z, \zeta)=1+\zeta_{1} z^{-1}+\ldots+\zeta_{\tilde{n}} z^{-\tilde{n}}$ and $B(z, \zeta)=z^{-1}\left(\zeta_{\tilde{n}+1}+\zeta_{\tilde{n}+2} z^{-1}+\ldots \zeta_{2 \tilde{n}} z^{-(\tilde{n}-1)}\right)$. As in Section 2.1, all these parameters are rational functions of $p$ (see (3)).

Defining $Z_{\text {den }}(p(t))=\left(\zeta_{1}(p(t)), \ldots, \zeta_{\tilde{n}}(p(t))\right)$ and $Z_{\text {num }}(p(t))=$ $\left(\zeta_{\tilde{n}+1}(p(t)), \ldots, \zeta_{2 \tilde{n}}(p(t))\right)$, we can propose the following state-space representation in canonical form for (A1):

$$
\begin{aligned}
& x(t+1)=\left(\frac{-Z_{\text {den }}(p(t))}{\left(I_{\tilde{n}-1} \mid 0\right)}\right) x(t)+\left(\frac{1}{0}\right) u(t) \\
& \breve{y}(t)=Z_{\text {num }}(p(t)) x(t)
\end{aligned}
$$

It is clear that the coefficients of the state-space matrices in (A2) are all rational in $p$. Consequently, this state-space representation can be used as a starting point for the LPI and LPV control design methods ${ }^{16}$.

${ }^{16}$ Note that $[13,29]$ are continuous-time control design methods. However, we can easily transform (A2) into a 
It is important to note that, as mentioned in [31], the representations (A1) and (A2) are only fully equivalent for scheduling sequences $p \in \mathbf{P}$ (i.e. in the case considered for LPI control). When $p \in \bar{P}$, the trajectories of (A1) and (A2) can be slightly different. Consequently, to be fully rigorous in the LPV situation, we should assume that the true LPV system and its model have the structure (A2) and that this structure is used for control design. Note nevertheless that, even if the LPV system is described by (A2) and not by (A1), this does not change the identification procedure of Section 2.2 since the LTI systems (8) corresponding to all operating points $\mathbf{p}_{\mathbf{m}} \in \mathcal{P}$ are fully identical for (A1) and (A2).

\section{Appendix B. Proof of Proposition 1}

Proposition 1 is a classical result in the literature of the so-called separation of graphs theory $[28,18,25]$. However, we give the proof for the sake of completeness. Let us thus suppose that there exist $A(\omega) \in \mathcal{A}$ and $\gamma<\infty$ such that (35) holds and let us first prove that this implies the stability of $\mathcal{F}(M(z), \Delta)$ for all $\Delta \in \Delta$. For this purpose, let us split $M$ in (29) into:

$$
\left(\begin{array}{c}
q \\
y
\end{array}\right)=\left(\begin{array}{cc}
M_{q s}(z) & M_{q w}(z) \\
M_{y s}(z) & M_{y w}(z)
\end{array}\right)\left(\begin{array}{c}
s \\
w
\end{array}\right)
$$

and let us observe that if there exist a finite $\gamma$ and a frequency-dependent matrix $A(\omega) \in \mathcal{A}$ such that (35) holds, this implies that, for the same $A(\omega) \in \mathcal{A}$ :

$$
\left(\begin{array}{c}
M_{q s}\left(e^{j \omega}\right) \\
I
\end{array}\right)^{*} A(\omega)\left(\begin{array}{c}
M_{q s}\left(e^{j \omega}\right) \\
I
\end{array}\right)<0 \quad \forall \omega
$$

Since the considered uncertainty set $\Delta$ is convex and contains 0 , the latter is a sufficient condition for the stability of $\mathcal{F}(M(z), \Delta)$ for all $\Delta \in \boldsymbol{\Delta}$ (see, e.g., [25]).

Let us now prove that the existence of $A(\omega) \in \mathcal{A}$ and $\gamma<\infty$ such that (35) holds implies (36). Condition (35) implies that:

$$
\frac{1}{2 \pi} \int_{-\pi}^{\pi} g^{*}\left(e^{j \omega}\right)\left(\begin{array}{c}
M\left(e^{j \omega}\right) \\
I
\end{array}\right)^{*} \mathcal{B}\left(\begin{array}{c}
M\left(e^{j \omega}\right) \\
I
\end{array}\right) g\left(e^{j \omega}\right) d \omega<0
$$

with $g\left(e^{j \omega}\right)=\left(s\left(e^{j \omega}\right), w\left(e^{j \omega}\right)\right)^{T}$ a vector containing the Fourier transform of signals $s(t)$ and $w(t)$ in $\mathcal{L}_{2}$. Since $\mathcal{F}(M(z), \Delta)$ is stable for all $\Delta \in \boldsymbol{\Delta}$, (B3) holds in particular for the signals $s(t)$ and $w(t)$ corresponding to any trajectory of $\mathcal{F}(M(z), \Delta)$ induced by signals $w(t) \in \mathcal{L}_{2}$ when $\Delta$ satisfies $\Delta \in \boldsymbol{\Delta}$. Since $M$ is stable, we can expand (B3) using (29):

$$
\begin{aligned}
& \frac{1}{2 \pi} \int_{-\pi}^{\pi}\left(\begin{array}{c}
q\left(e^{j \omega}\right) \\
s\left(e^{j \omega}\right)
\end{array}\right)^{*} A(\omega)\left(\begin{array}{c}
q\left(e^{j \omega}\right) \\
s\left(e^{j \omega}\right)
\end{array}\right) d \omega+\ldots \\
& \left.\ldots .+\frac{1}{2 \pi} \int_{-\pi}^{\pi}\left(\begin{array}{c}
y\left(e^{j \omega}\right) \\
w\left(e^{j \omega}\right)
\end{array}\right)\right)^{*} \operatorname{diag}(1,-\gamma)\left(\begin{array}{c}
y\left(e^{j \omega}\right) \\
w\left(e^{j \omega}\right)
\end{array}\right) d \omega<0
\end{aligned}
$$

Denoting the first integral in the left hand side of (B4) by $\mathcal{X}_{A}$ and using Parseval's theorem on (21), (B4) is equivalent with:

$$
\mathcal{X}_{A}+\|y\|_{2}^{2}<\gamma\|w\|_{2}^{2}
$$

continuous model rational in $p$ and the continuous-time controller can also be transformed into a discrete-time controller which is rational in $p$. 
Consequently, if there exist $A(\omega) \in \mathcal{A}$ and $\gamma$ such that (35) holds, this implies that, for the same $A(\omega)$ and $\gamma$, (B5) also holds for any trajectory $q, s, y, w$ of the systems $\mathcal{F}(M(z), \Delta)$ with $\Delta \in \boldsymbol{\Delta}$. Observe now that, by virtue of the fact that the frequencydependent matrix $A(\omega)$ lies in the set of multipliers associated to $\boldsymbol{\Delta}, \mathcal{X}_{A} \geq 0$ for the signals $(q, s)^{T}$ in any trajectory of the systems $\mathcal{F}(M(z), \Delta)$ with $\Delta \in \boldsymbol{\Delta}$. Consequently, $\|y\|_{2}^{2}<\gamma\|w\|_{2}^{2}$ for any trajectory of the systems $\mathcal{F}(M(z), \Delta)$ with $\Delta \in \boldsymbol{\Delta}$, which is equivalent to (36).

\section{Appendix C. Proof of Proposition 2}

Let us first split the first equation of (29):

$$
\left(\begin{array}{c}
s_{p} \\
s_{\delta \theta}
\end{array}\right)=\operatorname{bdiag}\left(p I_{n_{p}}, I_{n_{\theta}} \otimes \delta \theta\right)\left(\begin{array}{c}
q_{p} \\
q_{\delta \theta}
\end{array}\right)
$$

with $s_{p}(t) \triangleq\left(p(t) I_{n_{p}}\right) q_{p}(t)$ and $s_{\delta \theta}(t) \triangleq\left(I_{n_{\theta}} \otimes \delta \theta\right) q_{\delta \theta}(t)$.

With the block-diagonal structure of $A(\omega)$ given in Proposition 2 and (C1), we have that:

$$
\frac{1}{2 \pi} \int_{-\pi}^{\pi}\left(\begin{array}{c}
q\left(e^{j \omega}\right) \\
s\left(e^{j \omega}\right)
\end{array}\right)^{*} A(\omega)\left(\begin{array}{c}
q\left(e^{j \omega}\right) \\
s\left(e^{j \omega}\right)
\end{array}\right) d \omega=\mathcal{I}_{p}+\mathcal{I}_{\delta \theta}
$$

with

$$
\begin{aligned}
& \mathcal{I}_{p}=\frac{1}{2 \pi} \int_{-\pi}^{\pi} g_{p}^{*}\left(e^{j \omega}\right)\left(\begin{array}{ll}
A_{11, p}(\omega) & A_{12, p}(\omega) \\
A_{12, p}^{*}(\omega) & A_{22, p}(\omega)
\end{array}\right) g_{p}\left(e^{j \omega}\right) d \omega \\
& \mathcal{I}_{\delta \theta}=\frac{1}{2 \pi} \int_{-\pi}^{\pi} g_{\delta \theta}^{*}\left(e^{j \omega}\right)\left(\begin{array}{ll}
A_{11, \delta \theta}(\omega) & A_{12, \delta \theta}(\omega) \\
A_{12, \delta \theta}^{*}(\omega) & A_{22, \delta \theta}(\omega)
\end{array}\right) g_{\delta \theta}\left(e^{j \omega}\right) d \omega
\end{aligned}
$$

with $g_{p}\left(e^{j \omega}\right)=\left(q_{p}\left(e^{j \omega}\right) s_{p}\left(e^{j \omega}\right)\right)^{T}$ and $g_{\delta \theta}\left(e^{j \omega}\right)=\left(q_{\delta \theta}\left(e^{j \omega}\right) s_{\delta \theta}\left(e^{j \omega}\right)\right)^{T}$.

In order to prove the proposition, we will prove that

(1) for any matrices $A_{i j, p}(\omega)(i, j=1,2)$ as parametrized in the proposition, the integral $\mathcal{I}_{p}$ is larger than 0 for the Fourier transform $g_{p}\left(e^{j \omega}\right)$ of all $\left(q_{p}, s_{p}\right)^{T}$ in $\mathcal{G}_{\bar{P}}$

$$
\begin{aligned}
\mathcal{G}_{\bar{P}}=\{ & \left(\begin{array}{c}
q_{p} \\
s_{p}
\end{array}\right) \in \mathcal{L}_{2} \mid s_{p}(t)=\left(p I_{n_{p}}\right) q_{p}(t) \ldots \\
& \left.\ldots \text { with } q_{p} \in \mathcal{L}_{2} \text { and } p \in \bar{P}\right\} .
\end{aligned}
$$

(2) for any matrices $A_{i j, \delta \theta}(\omega)(i, j=1,2)$ as parametrized in the proposition, the integral $\mathcal{I}_{\delta \theta}$ is larger than 0 for the Fourier transform $g_{\delta \theta}\left(e^{j \omega}\right)$ of all $\left(q_{\delta \theta}, s_{\delta \theta}\right)^{T}$ in $\mathcal{G}_{U}$

$$
\begin{aligned}
\mathcal{G}_{U}=\{ & \left(\begin{array}{c}
q_{\delta \theta} \\
s_{\delta \theta}
\end{array}\right) \in \mathcal{L}_{2} \mid s_{\delta \theta}(t)=\left(I_{n_{\theta}} \otimes \delta \theta\right) q_{\delta \theta}(t) \ldots \\
& \left.\ldots \text { with } q_{\delta \theta} \in \mathcal{L}_{2} \text { and } \delta \theta \in U\right\} .
\end{aligned}
$$

Let us start with item (2). Since $\delta \theta$ is time-invariant, we have that $s_{\delta \theta}\left(e^{j \omega}\right)=$ $\left(I_{n_{\theta}} \otimes \delta \theta\right) q_{\delta \theta}\left(e^{j \omega}\right)$. Consequently, we can rewrite $\mathcal{I}_{\delta \theta}$ as follows:

$$
\mathcal{I}_{\delta \theta}=\frac{1}{2 \pi} \int_{-\pi}^{\pi} q_{\delta \theta}^{*}\left(e^{j \omega}\right) \mathcal{K}(\omega) q_{\delta \theta}\left(e^{j \omega}\right) d \omega
$$




$$
\mathcal{K}(\omega)=\left(\begin{array}{c}
I_{n_{\theta}} \\
I_{n_{\theta}} \otimes \delta \theta
\end{array}\right)^{T}\left(\begin{array}{cc}
A_{11, \delta \theta}(\omega) & A_{12, \delta \theta}(\omega) \\
A_{12, \delta \theta}^{*}(\omega) & A_{22, \delta \theta}(\omega)
\end{array}\right)\left(\begin{array}{c}
I_{n_{\theta}} \\
I_{n_{\theta}} \otimes \delta \theta
\end{array}\right)
$$

It is clear that, if $\mathcal{K}(\omega) \geq 0$ at all $\omega, \mathcal{I}_{\delta \theta} \geq 0$ whatever $q_{\delta \theta} \in \mathcal{L}_{2}$. Item (2) will therefore be proven if we show that, for any matrices $A_{i j, \delta \theta}(\omega)(i, j=1,2)$ having the parametrization in the statement of the proposition, $\mathcal{K}(\omega) \geq 0$ for all $\delta \theta \in U$ and that this holds true whatever $\omega$. That the latter holds is a direct consequence of Proposition 2 in [5]. Indeed, whatever $\omega$, we have the following identities for all $A_{12, \delta \theta}(\omega), \tilde{A}(\omega)$ and $\tilde{B}(\omega)$ as parametrized in the proposition:

$$
\begin{gathered}
A_{12, \delta \theta}(\omega)\left(I_{n_{\theta}} \otimes \delta \theta\right)+\left(I_{n_{\theta}} \otimes \delta \theta\right)^{T} A_{12, \delta \theta}^{*}(\omega)=0 \\
\left(I_{n_{\theta}} \otimes \delta \theta\right)^{T} j \tilde{A}(\omega)\left(I_{n_{\theta}} \otimes \delta \theta\right)=0 \\
\left(I_{n_{\theta}} \otimes \delta \theta\right)^{T} \tilde{B}(\omega)\left(I_{n_{\theta}} \otimes \delta \theta\right)=0
\end{gathered}
$$

Consequently:

$$
\mathcal{K}(\omega)=\left(1-\delta \theta^{T} \frac{P_{\theta}^{-1}}{\chi} \delta \theta\right) A_{0}(\omega)
$$

Recalling that $A_{0}(\omega)$ can take any values as long as it is positive definite at each $\omega$, we see that, whatever $\omega, \mathcal{K}(\omega)$ is indeed a positive semi-definite matrix for all $\delta \theta \in U$.

Let us now consider item (1). Since $p$ is time-varying, we need to prove this item differently [25]. Using the frequency-independent parametrization of $A_{i j, p}(i, j=1,2)$ and recalling that $s_{p}(t)=\left(p(t) I_{n_{p}}\right) q_{p}(t)$, we can use Parseval's theorem to rewrite $\mathcal{I}_{p}$ successively as follows:

$$
\begin{gathered}
\mathcal{I}_{p}=\sum_{t=0}^{\infty}\left(\begin{array}{c}
q_{p}(t) \\
s_{p}(t)
\end{array}\right)^{T}\left(\begin{array}{cc}
p_{\max }^{2} \mathcal{S} & \mathcal{G} \\
\mathcal{G}^{*} & -\mathcal{S}
\end{array}\right)\left(\begin{array}{c}
q_{p}(t) \\
s_{p}(t)
\end{array}\right) \\
\mathcal{I}_{p}=\sum_{t=0}^{\infty} q_{p}^{T}(t) \underbrace{\left(\begin{array}{c}
I_{n_{p}} \\
p(t) I_{n_{p}}
\end{array}\right)^{T}\left(\begin{array}{cc}
p_{\max }^{2} \mathcal{S} & \mathcal{G} \\
\mathcal{G}^{*} & -\mathcal{S}
\end{array}\right)\left(\begin{array}{c}
I_{n_{p}} \\
p(t) I_{n_{p}}
\end{array}\right)}_{=\mathcal{X}(t)} q_{p}(t)
\end{gathered}
$$

It is clear that, if $\mathcal{X}(t) \geq 0$ at all $t, \mathcal{I}_{p} \geq 0$ whatever $q_{p} \in \mathcal{L}_{2}$. Item (1) will therefore be proven if we show that, for any matrices $\mathcal{S}$ and $\mathcal{G}$ having the structure given in the statement of the proposition, $\mathcal{X}(t) \geq 0$ for all $p \in \bar{P}$ and that this holds true whatever $t$. That the latter holds is easily proven. Indeed, using the fact that $p(t)$ is a scalar signal, we observe that, whatever $t$,

$$
\mathcal{X}(t)=\left(p_{\text {max }}^{2}-p^{2}(t)\right) \mathcal{S}+p(t)\left(\mathcal{G}+\mathcal{G}^{*}\right) .
$$

Using the facts that $\mathcal{G}$ is skew-symmetric, $\mathcal{X}(t)$ reduces to:

$$
\mathcal{X}(t)=\left(p_{\text {max }}^{2}-p^{2}(t)\right) \mathcal{S}
$$

Recalling (19) and (1) and the fact that $\mathcal{S}$ is restricted to be positive definite, we conclude that, whatever $t, \mathcal{X}(t)$ is indeed positive semi-definite for all $p(t) \in \bar{P}$. 


\section{Appendix D. Proof of Proposition 3}

The proof follows the same line as the one of Proposition 2. The reasoning starts also with $(\mathrm{C} 2)$ where $\mathcal{I}_{p}$ and $\mathcal{I}_{\delta \theta}$ have the expressions given in (C3). In order to prove Proposition 3, we will prove that:

(1) for any matrices $A_{i j, p}(\omega)(i, j=1,2)$ as parametrized in the proposition, the integral $\mathcal{I}_{p}$ is larger than 0 for the Fourier transform $g_{p}\left(e^{j \omega}\right)$ of all $\left(q_{p}, s_{p}\right)^{T}$ in $\mathcal{G}_{\mathbf{P}}$

$$
\begin{aligned}
\mathcal{G}_{\mathbf{P}}=\{ & \left(\begin{array}{c}
q_{p} \\
s_{p}
\end{array}\right) \in \mathcal{L}_{2} \mid s_{p}(t)=\left(\mathbf{p} I_{n_{p}}\right) q_{p}(t) \ldots \\
& \left.\ldots . . \text { with } q_{p} \in \mathcal{L}_{2} \text { and } \mathbf{p} \in \mathcal{P}\right\} .
\end{aligned}
$$

(2) for any matrices $A_{i j, \delta \theta}(\omega)(i, j=1,2)$ as parametrized in the proposition, the integral $\mathcal{I}_{\delta \theta}$ is larger than 0 for the Fourier transform $g_{\delta \theta}\left(e^{j \omega}\right)$ of all $\left(q_{\delta \theta}, s_{\delta \theta}\right)^{T}$ in $\mathcal{G}_{U}($ see $(\mathrm{C} 4))$.

The proof of item (2) is the same as in Appendix C. Let us thus prove item (1). In this case, since $\mathbf{p}$ is time-invariant, we have that $s_{p}\left(e^{j \omega}\right)=\left(\mathbf{p} I_{n_{p}}\right) q_{p}\left(e^{j \omega}\right)$. This yields the following relation for $\mathcal{I}_{p}$ :

$$
\begin{gathered}
\mathcal{I}_{p}=\int_{-\pi}^{\pi} q_{p}^{*}\left(e^{j \omega}\right) \mathcal{X}(\omega) q_{p}^{*}\left(e^{j \omega}\right) d \omega \\
\mathcal{X}(\omega)=\left(\begin{array}{c}
I_{n_{p}} \\
\mathbf{p} I_{n_{p}}
\end{array}\right)^{T}\left(\begin{array}{cc}
p_{\max }^{2} \mathcal{S}(\omega) & \mathcal{G}(\omega) \\
\mathcal{G}^{*}(\omega) & -\mathcal{S}(\omega)
\end{array}\right)\left(\begin{array}{c}
I_{n_{p}} \\
\mathbf{p} I_{n_{p}}
\end{array}\right)
\end{gathered}
$$

It is clear that, if $\mathcal{X}(\omega) \geq 0$ at all $\omega, \mathcal{I}_{p} \geq 0$ whatever $q_{p} \in \mathcal{L}_{2}$. Item (1) will therefore be proven if we show that, for any matrices $\mathcal{S}(\omega)$ and $\mathcal{G}(\omega)$ having the parametrization in the statement of the proposition, $\mathcal{X}(\omega) \geq 0$ for all $\mathbf{p} \in \mathcal{P}$ and that this holds true for all $\omega$. That the latter holds follows from similar arguments than in Appendix C. Indeed, using the fact that $\mathbf{p}$ is a scalar, we observe that, whatever $\omega$,

$$
\mathcal{X}(\omega)=\left(p_{\max }^{2}-\mathbf{p}^{2}\right) \mathcal{S}(\omega)+\mathbf{p}\left(\mathcal{G}(\omega)+\mathcal{G}^{*}(\omega)\right)
$$

Using the facts that $\mathcal{G}(\omega)$ is skew-symmetric, $\mathcal{X}(\omega)$ reduces to:

$$
\mathcal{X}(\omega)=\left(p_{\max }^{2}-\mathbf{p}^{2}\right) \mathcal{S}(\omega)
$$

Recalling (1) and the fact that $\mathcal{S}(\omega)$ is restricted to be positive definite, we conclude that, whatever $\omega, \mathcal{X}(\omega)$ is indeed positive semi-definite for all $\mathbf{p} \in \mathcal{P}$.

\section{Appendix E. Proof of Proposition 5}

This proposition is also a classical result of the separation of graphs theory, but we give the proof for completeness. Let us consider a given frequency $\omega$ and let us first 
observe that if there exist a finite $\gamma(\omega)$ and a matrix $A(\omega)$ having the structure of Proposition 3 such that (38) holds, this implies that, for the same $A(\omega)$,

$$
\left(\begin{array}{c}
M_{q s}\left(e^{j \omega}\right) \\
I
\end{array}\right)^{*} A(\omega)\left(\begin{array}{c}
M_{q s}\left(e^{j \omega}\right) \\
I
\end{array}\right)<0
$$

using the notations in (B1). That (E1) holds for a matrix $A(\omega)$ having the structure of Proposition 3 is a sufficient condition to ensure that $\operatorname{det}\left(I-M_{q s}\left(e^{j \omega}\right) \Delta\right) \neq 0$ for all $\Delta \in \Delta_{\mathbf{P}}$ [28]. One consequence of the latter is that the LFT relation (29) evaluated at $\omega$ is well-posed for all $\Delta \in \Delta_{\mathbf{P}}$.

Using this well-posed relation at $\omega$, let us now observe that, whatever $\Delta \in \boldsymbol{\Delta}_{\mathbf{P}}$, the complex number $\mathcal{F}\left(M\left(e^{j \omega}\right), \Delta\right)$ is equal to $y\left(e^{j \omega}\right)$ in the following expression:

$$
\begin{aligned}
& s\left(e^{j \omega}\right)=\overbrace{b \operatorname{diag}\left(\mathbf{p} I_{n_{p}}, I_{n_{\theta}} \otimes \delta \theta\right)}^{=\Delta} q\left(e^{j \omega}\right) \\
& \left(\begin{array}{c}
q\left(e^{j \omega}\right) \\
y\left(e^{j \omega}\right)
\end{array}\right)=M\left(e^{j \omega}\right) \underbrace{\left(\begin{array}{c}
s\left(e^{j \omega}\right) \\
1
\end{array}\right)}_{=g\left(e^{j \omega}\right)}
\end{aligned}
$$

The fact that (E2) is well-posed for all $\Delta \in \Delta_{\mathbf{P}}$ implies that, for each $\Delta \in \Delta_{\mathbf{P}}$, one can determine finite complex numbers/vectors $q\left(e^{j \omega}\right), s\left(e^{j \omega}\right)$ and $y\left(e^{j \omega}\right)=\mathcal{F}\left(M\left(e^{j \omega}\right), \Delta\right)$ satisfying/solving relation (E2).

To prove Proposition 5, note first that condition (38) implies:

$$
g^{*}\left(e^{j \omega}\right)\left(\begin{array}{c}
M\left(e^{j \omega}\right) \\
I
\end{array}\right)^{*} \mathcal{B}\left(\begin{array}{c}
M\left(e^{j \omega}\right) \\
I
\end{array}\right) g\left(e^{j \omega}\right)<0
$$

with $g\left(e^{j \omega}\right)$ as defined in (E2). We can expand (E3) using (E2):

$$
\begin{aligned}
& \left(\begin{array}{c}
q\left(e^{j \omega}\right) \\
s\left(e^{j \omega}\right)
\end{array}\right)^{*} A(\omega)\left(\begin{array}{c}
q\left(e^{j \omega}\right) \\
s\left(e^{j \omega}\right)
\end{array}\right)+\ldots \\
& \ldots .+\left(\begin{array}{c}
y\left(e^{j \omega}\right) \\
1
\end{array}\right)^{*} \operatorname{diag}(1,-\gamma(\omega))\left(\begin{array}{c}
y\left(e^{j \omega}\right) \\
1
\end{array}\right)<0
\end{aligned}
$$

Denoting the first term in the left hand side of (E4) by $\mathcal{X}_{A}(\omega),(\mathrm{E} 4)$ yields

$$
\mathcal{X}_{A}(\omega)+\left|y\left(e^{j \omega}\right)\right|^{2}<\gamma(\omega)
$$

Consequently, if there exists $\gamma(\omega)>0$ and a matrix $A(\omega)$ having the structure given in Proposition 3 such that (38) holds, this implies that, for the same $A(\omega)$ and $\gamma(\omega)$, (E5) holds for all $q\left(e^{j \omega}\right), s\left(e^{j \omega}\right)$ and $y\left(e^{j \omega}\right)$ that can be obtained by solving relation (E2) for a given $\Delta \in \boldsymbol{\Delta}_{\mathbf{P}}$.

Using the notations introduced in Appendices $\mathrm{C}$ and $\mathrm{D}, \mathcal{X}_{A}(\omega)$ can be rewritten as:

$$
\mathcal{X}_{A}(\omega)=q_{p}^{*}\left(e^{j \omega}\right) \mathcal{X}(\omega) q_{p}\left(e^{j \omega}\right)+q_{\delta \theta}^{*}\left(e^{j \omega}\right) \mathcal{K}(\omega) q_{\delta \theta}\left(e^{j \omega}\right)
$$

with $q=\left(q_{p}, q_{\delta \theta}\right)^{T}$. Since we show in Appendix D, that, for the matrices $A(\omega)$ having the structure of Proposition 3, both $\mathcal{K}(\omega)$ and $\mathcal{X}(\omega)$ are positive semi-definite matrices for all $\Delta \in \boldsymbol{\Delta}_{\mathbf{P}}$, we can conclude that $\mathcal{X}_{A}(\omega) \geq 0$ for all $q\left(e^{j \omega}\right)$ and $s\left(e^{j \omega}\right)$ satisfying 
relation (E2) with a certain $\Delta \in \boldsymbol{\Delta}_{\mathbf{P}}$. Consequently, we have that $\left|y\left(e^{j \omega}\right)\right|^{2}<\gamma(\omega)$ for all $y\left(e^{j \omega}\right)$ satisfying relation (E2) with $\Delta \in \boldsymbol{\Delta}_{\mathbf{P}}$, which is equivalent to:

$$
\left|\mathcal{F}\left(M\left(e^{j \omega}\right), \Delta\right)\right|^{2}<\gamma(\omega) \quad \forall \Delta \in \Delta_{\mathbf{P}}
$$

With this, we have thus proven that $J_{w c}^{u b}(\omega)$ is (at least) an upper bound of (39). The second part of the proposition is a direct consequence of the facts i) that, due to the structure of $\mathcal{A}_{\mathbf{P}}$ in Proposition 3, we can independently choose the value at each $\omega$ of the frequency-dependent matrix lying in $\mathcal{A}_{\mathbf{P}}$ that has to be determined in the optimization problem of Proposition 4 pertaining to the LPI situation and ii) that, if there exists $\gamma(\omega)>0$ and a matrix $A(\omega)$ having the structure given in Proposition 3 such that (38) holds, then, for the same $A(\omega),(38)$ also holds for any $\gamma^{*} \geq \gamma(\omega)$.

Remark. Since $\boldsymbol{\Delta}_{\mathbf{P}}$ is a time-invariant uncertainty set, the stability of $\mathcal{F}(M(z), \Delta)$ for all $\Delta \in \boldsymbol{\Delta}_{\mathbf{P}}$ can be verified by checking that, at all $\omega, \operatorname{det}\left(I-M_{q s}\left(e^{j \omega}\right) \Delta\right) \neq 0$ for all $\Delta \in \Delta_{\mathbf{P}}$ [36]. It is clear from the first part of Proposition 5 that this will be verified if $J_{w c}^{u b}(\omega)$ is finite at all frequencies. 